\title{
miR-135a-5p Functions as a Glioma Proliferation Suppressor by Targeting Tumor Necrosis Factor Receptor-Associated Factor 5 and Predicts Patients' Prognosis
}

Wenjun Luo, ${ }^{* \dagger \ddagger}$ Cuiyun Sun, ${ }^{* \dagger \ddagger}$ Junhu Zhou, ${ }^{\dagger+\delta}$ Qian Wang, ${ }^{* \dagger}$ Lin Yu, ${ }^{\oplus}$ Xiu-Wu Bian, Xuexia Zhou, ${ }^{\star \dagger \ddagger}$ Dan Hua, ${ }^{* \dagger \ddagger}$

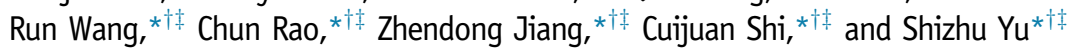

From the Department of Neuropathology* and the Laboratory of Neuro-Oncology, ${ }^{\S}$ Tianjin Neurological Institute, Tianjin Medical University General Hospital, Tianjin; the Tianjin Key Laboratory of Injuries, Variations and Regeneration of the Nervous System, ${ }^{\dagger}$ Tianjin; the Key Laboratory of Post-Trauma Neuro-Repair and Regeneration in Central Nervous System, ${ }^{\ddagger}$ Ministry of Education, Tianjin; the Department of Biochemistry and Molecular Biology, ${ }^{\top}$ School of Basic Medical Sciences of Tianjin Medical University, Tianjin; and the Institute of Pathology and Southwest Cancer Center," Southwest Hospital, Third Military Medical University, Chongqing, People's Republic of China

\author{
Accepted for publication \\ August 31, 2018. \\ Address correspondence to \\ Shizhu Yu, M.D., Ph.D., or \\ Cuijuan Shi, Ph.D., Department \\ of Neuropathology, Tianjin \\ Neurological Institute, Tianjin \\ Medical University General \\ Hospital, Number 154 \\ Anshan Rd, Tianjin 300052, \\ China. E-mail: tjyushizhu@ \\ 163.com or cshi@tmu.edu.cn.
}

\begin{abstract}
miR-135a-5p has been reported as a tumor suppressor in several extracranial tumors. However, its exact roles in gliomagenesis and relevance to the patients' prognoses are largely unknown. Herein, we detected the miR-135a-5p and tumor necrosis factor receptor-associated factor 5 (TRAF5) levels in 120 human glioma specimens and 20 nontumoral brain tissues; we found the miR-135a-5p level decreased, whereas the TRAF5 level increased, with the elevation of glioma grade. Their labeling indexes were inversely correlated with each other and showed strong negative (miR-135a-5p) and positive (TRAF5) correlation with the Ki-67 index. Cox regression demonstrated that both of their expression levels were independent survival predictors, whereas Kaplan-Meier analysis showed that subgrouping the glioma patients according to their levels could perfectly reflect the patients' prognoses regardless of the similarities in pathologic, molecular, and clinical features. In the following in vitro and in vivo studies, it was demonstrated that miR-135a-5p induced $\mathrm{G}_{1}$ arrest and inhibited the proliferation of glioma cells by targeting TRAF5 and subsequently blocking AKT phosphorylation as well as C-Myc and cyclin D1 expression. These effects could be reversed by TRAF5 overexpression and simulated by specific TRAF5 silencing. This study highlights the importance of miR-135a-5p and TRAF5 in gliomagenesis and progression and implies their potential prognostic and therapeutic values in malignant glioma. (Am J Pathol 2019, 189: 162-176; https://doi.org/10.1016/j.ajpath.2018.08.019)
\end{abstract}

Gliomas are the most common primary brain tumors. ${ }^{1,2}$ High-grade gliomas, especially glioblastoma (GBM), are highly recurrent and lethal because of their rampant growth and invasion, which makes radical resection almost clinically unfeasible. ${ }^{3}$ Despite the benefits of alkylating agents, such as temozolomide, and the advances in neurosurgery, the patients' prognoses remain poor, with a median survival of no more than 20 months. $^{4}$ In recent years, the great efforts in biomarker screening and therapeutic target selection have been constantly improving the diagnosis, prognosis, and therapy of gliomas.
Supported by National Natural Science Foundation of China grants 81872061 (C.S.), 81402050 (C.S.), 81502166 (X.Z.), and 81672592 (L.Y.); Program of Science and Technology of Tianjin Municipality grants 15JCYBJC49900 (C.S.), 15JCZDJC34600 (Q.W.), 16JCQNJC13400 (D.H.), and 17JCYBJC27100 (X.Z.); Program of Tianjin Municipal Health Bureau grant 15KJ147 (Q.W.); Foundation of Tianjin Medical University and General Hospital grants 2015KYZQ11 (D.H.), ZYYFY2014038 (X.Z.), and ZYYFY2015032 (D.H.); and Tianjin Medical University General Hospital Junior Technical Backbone Training Project 2016 (C.S.). W.L., C.S., and J.Z. contributed equally to this work.

Disclosures: None declared. 
The biological functions and prognostic values of some miRNAs have been demonstrated in various kinds of tumors. ${ }^{6,7,9-12}$ However, the effects of miR-135a-5p, a miR-135 family member, is somewhat complicated, because it may exert opposite roles in different tumors. miR-135a-5p has been confirmed as a tumor suppressor in non-small-cell lung cancer, ${ }^{13}$ gastric cancer, ${ }^{14}$ pancreatic carcinoma, ${ }^{15}$ and metastatic prostate cancer. ${ }^{16}$ However, in colorectal cancer, higher miR-135a-5p is often associated with more malignant phenotypes. ${ }^{17}$ These findings prompted us to investigate this miRNA specifically in gliomas.

As a potent activator of the mitogen-activated protein kinase and NF- $\kappa B$ pathway, tumor necrosis factor receptor-associated factor 5 (TRAF5) participates in the onset and progression of several tumors. ${ }^{18-20}$ This conclusion is further supported by the findings that miR-26b inhibits melanoma cell proliferation and numb-like protein inhibits glioma cell migration/invasion by directly targeting TRAF5. ${ }^{18,19}$ Bioinformatics predicts that TRAF5 is a candidate target of miR-135a-5p. However, to the best of our knowledge, whether this miRNA suppresses glioma cell proliferation by silencing TRAF5 has not been reported.

In this study, we reported, for the first time, that the low expression of miR-135a-5p is responsible for the abnormal increase of TRAF5 in glioma cells. Introducing exogenous miR-135a-5p effectively suppressed TRAF5 expression and thereby obstructed the proliferation of glioma cells. Our findings also indicated the prognostic values of these molecules as independent predictors and the usefulness of them in glioma therapy.

\section{Materials and Methods}

\section{Tissue Samples and Clinical Data}

The surgical specimens of human gliomas and 20 nontumoral control brain tissues were collected from Tianjin Medical University General Hospital (Tianjin, China) with written consents. For the glioma specimens, IDHI/2 gene mutations were detected by Sanger sequencing, and the statuses of chromosome arms $1 \mathrm{p}$ and $19 \mathrm{q}$ were detected by either fluorescence in situ hybridization or comparative genomic hybridization. Then, the diagnoses were updated according to the 2016 World Health Organization classification of central nervous system tumors, ${ }^{2}$ and only 120 patients with astrocytic gliomas without $1 \mathrm{p} / 19 \mathrm{q}$ codeletion were enrolled in the present study. All the glioma patients with complete information were followed up since the date of operation to December 31,2013, with a follow-up time of 4.5 to 89 months. The clinical and pathologic features are summarized in Supplemental Table S1. This study was performed in accordance with the principles of the Declaration of Helsinki and approved by the Ethics Committee of Tianjin Medical University General Hospital.

The miRNA microarray expression data from the Chinese Glioma Genome Atlas (CGGA; http://www.cgga.org.cn, last accessed June 20, 2018) were used to further verify the expression change of miR-135a-5p and its interrelationship with patients' survival. Meanwhile, the data from the CGGA, The Cancer Genome Atlas (https:// cancergenome.nih.gov, last accessed June 20, 2018), and Oncomine (https://www.oncomine.org, last accessed June 20, 2018) were used for TRAF5 mRNA expression analysis and prognostic value assessment.

\section{ISH and IHC}

In situ hybridization (ISH) and immunohistochemistry (IHC) were performed as described previously. ${ }^{6}$ The locked nucleic acid-modified and digoxin-labeled oligonucleotide probe for miR-135a-5p and the corresponding scrambled control sequence were purchased from Exiqon (Vedbaek, Denmark) (Table 1). Mouse anti-human TRAF5 antibody and rabbit anti-human Ki-67 antibody were purchased from Santa Cruz Biotechnology (Dallas, TX) and Millipore (Billerica, MA), respectively. The ISH and IHC images were captured under a DM6000B microscope (Leica, Wetzlar, Germany), and the ratios [labeling index (LI) (\%)] of the positive cell number/the total cell number in five randomly selected $\times 400$ fields were calculated with Image Pro Plus 5.0 (Media Cybernetics, Rockville, MD).

\section{Cell Lines and Cell Culture}

Human glioblastoma cell lines U251 and U87MG were purchased from the China Academic Sinica Cell Repository (Shanghai, China) and ATCC (Manassas, VA), respectively. For patient-derived culture (primary GBM), fresh glioblastoma sample was minced in phosphate-buffered saline, digested by trypsin on a $37^{\circ} \mathrm{C}$ shaker for 20 minutes, filtered through nylon mesh filters, and washed with phosphatebuffered saline three times. Both the cell lines and primary GBM were maintained in Dulbecco's modified Eagle's medium (Gibco, Grand Island, NY) with 10\% fetal bovine serum (Biological Industries, Kibbutz Beit Haemek, Israel). For neurosphere culture, U87MG cells were cultivated in Dulbecco's modified Eagle's medium/F12 medium (Corning, Corning, NY) supplemented with $20 \mathrm{ng} / \mathrm{mL}$ epidermal growth factor (PeproTech, Rocky Hill, NJ), $20 \mathrm{ng} / \mathrm{mL}$ basic fibroblast growth factor (PeproTech), and B27 (Gibco). All the cells were incubated at $37^{\circ} \mathrm{C}$ in $5 \% \mathrm{CO}_{2}$ in a humidified incubator.

Table 1 The Probes of miR-135a-5p and Scr Used for ISH

\begin{tabular}{ll}
\hline Oligonucleotide probe & Sequence \\
\hline DIG-labeled LNA miR-135a-5p & $5^{\prime}$-TCACATAGGAATAAAA- \\
AGCCATA-3' & 5IG-labeled LNA Scr \\
& AATTAGG-3' \\
\hline
\end{tabular}

DIG, digoxin; ISH, in situ hybridization; LNA, locked nucleic acid; Scr, scrambled control sequence. 


\section{Stable Sub-Cell Line Establishment}

The miR-135a-5p-expressing lentivirus and the control (Con) lentivirus were purchased from Genechem (Shanghai, China). The TRAF5-expressing lentivirus was constructed and packaged by Applied Biological Materials (Vancouver, BC, Canada). The stable sub-cell lines of U251 (U251Con, -miR-135a-5p, and -miR-135a-5p+TRAF5), U87MG (U87MG-Con, -miR-135a-5p, and -miR-135a-5p+TRAF5), and primary GBM (primary GBM-Con, - miR-135a-5p, and -miR-135a-5p+TRAF5) were established by corresponding lentivirus infection. The expression efficiencies of the exogenous genes were confirmed by real-time quantitative RT-PCR (RT-qPCR) and Western blot analysis.

\section{Oligonucleotides and Cell Transfection}

The specific siRNAs of TRAF5 (siTRAF5\#1 and siTRAF5\#2) and the scrambled control (Ribobio, Guangzhou, China) (Table 2) were used to transfect U251 and U87MG cells with X-tremeGENE siRNA Transfection Reagent (Roche, Indianapolis, IN).

\section{RT-qPCR}

RT-qPCR was performed as previously described. ${ }^{21}$ miR-135a-5p was quantified by the Stem-Loop Detection Kit (GenePharma, Shanghai, China) using U6 as the internal control, and TRAF5 mRNA was quantified by the GoTaq qPCR Master Mix Kit (Promega, Fitchburg, WI) using $G A P D H$ as the internal control. The primers (Table 3) were synthesized by Invitrogen (Shanghai, China). The coarse data were then converted by $2^{-\Delta \Delta \mathrm{Ct}}$ transformation to represent the fold changes of the RNAs.

\section{Cell Proliferation Assays}

For 5-ethynyl-2'-deoxyuridine assays, cells $\left(5 \times 10^{3}\right.$ per well) were seeded into 96-well plates or poly-L-lysine/ laminin-coated 96-well plates (neurosphere culture). The stable sub-cell lines and the siRNA-transfected cells were stained 24 hours after seeding and 48 hours after transfection, respectively, with the Cell-Light EdU Apollo567 in Vitro Imaging kit (Ribobio), according to the

Table 2 The siRNAs and Scrambled Control Sequence

\begin{tabular}{ll}
\hline $\begin{array}{l}\text { dsRNA } \\
\text { oligonucleotide }\end{array}$ & Sequence \\
\hline TRAF5 siRNA & F: 5'-GGAUGUAAUGCCAAGGUUATT-3' \\
(siTRAF5\#1) & R: 5'-UAACCUUGGCAUUACAUCCTT-3' \\
TRAF5 siRNA & F: 5'-GGAGCACAUGCGUUUGGUUTT-3' \\
(siTRAF5\#2) & R: 5'-AACCAAACGCAUGUGCUCCTT-3' \\
Scrambled control & F: 5'-UUCUCCGAACGUGUCACGUTT-3' \\
sequence & R: 5'-ACGUGACACGUUCGGAGAATT-3' \\
\hline
\end{tabular}

dsRNA, double-stranded RNA; F, forward; $R$, reverse.
Table 3 Primers Used for miR-135a-5p and TRAF5 mRNA RT-qPCR Detections

\begin{tabular}{ll}
\hline Primer & Sequence \\
\hline miR-135a-5p & F: 5'-CCGGCGTATGGCTTTTTATTCC-3' \\
U6 & R: 5'-CAGTGCAGGGTCCGAGGT-3' \\
& F: 5'-CGCAAGGATGACACGCAAATTCG-3' \\
TRAF5 & R: 5'-CAGTGCAGGGTCCGAGGT-3' \\
& F: 5'-CCTACGGAAAGACCTGAAAGAGC-3' \\
GAPDH & R: 5'-GGGTATTCAGGACACAAGTTTTCC-3' \\
& F: 5'-TGCACCACCAACTGCTTAGC-3' \\
& R: 5'-GGCATGGACTGTGGTCATGAG-3' \\
\hline
\end{tabular}

$F$, forward; $R$, reverse; RT-qPCR, real-time quantitative RT-PCR.

manufacturer's instruction. For 3-(4, 5-dimethylthiazol-2yl)-5-(3-carboxymethoxyphenyl)-2-(4-sulfophenyl)-2H-tetrazolium (MTS) proliferation assays, cells $\left(1 \times 10^{3}\right.$ per well $)$ were cultivated for $24,48,72,96$, and 120 hours. At the indicated intervals, $20 \mu \mathrm{L}$ of Cell Titer $96 \mathrm{AQ}_{\text {euous }}$ One Solution Reagent (Promega) was added to each well and incubated for 2 hours at $37^{\circ} \mathrm{C}$. The absorbance at $490 \mathrm{~nm}$ was measured with a Synergy microplate reader (BioTek Instruments, Winooski, VT).

\section{Target Prediction and Dual-Luciferase Reporter Assay}

The candidate targets of miR-135a-5p were predicted using TargetScan version 7.0 (http://www.targetscan.org/vert_70, last accessed June 20,2018). As a member of the TRAF family, TRAF5 was focused on the basis of the site type, combination of the context score, context score percentile, and probability of conserved targeting. The cDNA fragment corresponding to the $3^{\prime}$-untranslated region (UTR) of TRAF5 (TRAF5-3'-UTR-wild type) and its mutant (TRAF5-3'-UTR-mutant) lacking the miR-135a-5p target sequence were synthesized by GenScript (Nanjing, China) and inserted into the multiple cloning site of the pEZX-MT01 vector (GeneCopoeia, Rockville, MD) downstream of the firefly luciferase reporter gene to construct the recombinant reporter plasmids wild type and mutant type. These plasmids were then used to transfect the Con and the miR-135a-5p overexpression sub-cell lines with X-tremeGENE HP DNA Transfection Reagent (Roche). Firefly and renilla luciferase activities were detected with the Dual-Luciferase Reporter Assay System (Promega). The results were presented as the firefly luciferase activities normalized against those of renilla.

\section{Western Blot Analysis}

Western blot analysis was performed as previously described. ${ }^{22}$ The mouse anti-human TRAF5 and cyclin D1 antibodies (Santa Cruz Biotechnology); rabbit anti-human phosphorylated AKT and AKT antibodies (CST, Boston, MA); rabbit anti-human c-Myc antibody (Bioworld, 
Nanjing, China); and mouse anti-human glyceraldehyde-3phosphate dehydrogenase antibody (Boster, Wuhan, China) were used as the primary antibodies.

\section{Flow Cytometry Assay}

The stable sub-cell lines and siRNA-transfected cells were harvested and fixed in $70 \%$ ethanol at $4^{\circ} \mathrm{C}$ overnight. The cells were incubated with $40 \mu \mathrm{g} / \mathrm{mL}$ propidium iodide (Sigma, St. Louis, MO) at room temperature for 30 minutes and analyzed with an Accuri C6 flow cytometer (BD, Franklin Lakes, NJ). The data were processed with ModFit LT software version 3.1 (Verity Software House, Topsham, ME).

\section{Tumor Xenograft Assays}

The animal experiments were approved by the Institutional Animal Care and Use Committee of Tianjin Medical University General Hospital. Six-week-old BALB/C athymic nude mice (National Laboratory Animal Center, Beijing, China) were anesthetized and intracranially injected with $5 \times 10^{5}$ U87MG sub-cell lines (U87MG-Con, -miR-135a$5 \mathrm{p}$, or -miR-135a-5p+TRAF5) and primary GBM (primary GBM-Con, -miR-135a-5p, and -miR-135a-5p+TRAF5). The growth of the xenografts was monitored with the IVIS Lumina Imaging System (PerkinElmer, Waltham, MA) 7, 14, 21, and 28 days after implantation. The mice were sacrificed at day 35, and the brains were collected for hematoxylin and eosin and IHC staining.

\section{Statistical Analysis}

Statistical analyses were performed using SPSS software version 21.0 (IBM, Chicago, IL). Data were presented as means $\pm \mathrm{SD}$. One-way analysis of variance, $t$-test, Pearson's correlation, Kaplan-Meier analysis, log-rank test, and Cox proportional hazards regression were used to analyze corresponding data. The medians of the miR-135a-5p and TRAF5 labeling indexes were used as cutoffs in survival analyses. Statistical significance was assigned at $P<0.05$. All experiments in vitro and in vivo were performed at least three times with triplicate samples.

\section{Results}

\section{Low miR-135a-5p Expression Predicts Poorer Prognoses of Glioma Patients}

ISH results showed that the expression level of miR-135a$5 \mathrm{p}$ was significantly lower in the gliomas than in the control brains $(P<0.001)$. Among the glioma groups, the LI of miR-135a-5p declined gradually with the elevation of the grade $(P<0.001)$ (Figure $1, \mathrm{~A}$ and $\mathrm{B})$ and showed an inverse correlation with the Ki-67 proliferation index $(r=-0.782, P<0.0001$ ) (Figure $1 \mathrm{C}$ and Supplemental
Figure S1, A and B). Kaplan-Meier analysis revealed that the disease-free survival (DFS) and overall survival (OS) of the patients with a high miR-135a-5p level were significantly longer than those of the patients with a low miR135a-5p level (DFS, $P<0.0001 ;$ OS, $P<0.0001$ ) (Figure 1D). These differences remained obvious even within the patients with the same tumor grade, same $I D H$ status, similar ages, and similar Karnofsky performance scores (DFS, $P<0.01$ to approximately $P<0.0001$; OS, $P<0.01$ to approximately $P<0.0001$ ) (Figure $1, \mathrm{E}-\mathrm{I}$, and Supplemental Figure S2). The above findings were further confirmed by the data from the CGGA (Supplemental Figure S3A). Furthermore, Cox regression demonstrated that miR-135a-5p was an independent predictor for DFS and OS of glioma patients (Table 4 and Supplemental Table S2). These results indicate the practical value of miR-135a-5p in prognosis assessment and imply its potential antiproliferation effect.

\section{miR-135a-5p Suppresses the Proliferation of Human Glioma Cells}

Prompted by the inverse correlation between miR-135a-5p LI and the Ki-67 index, miR-135a-5p-overexpressing U251 and U87MG sub-cell lines and the corresponding controls were established by recombinant lentivirus infection. miR135a-5p expression was monitored by RT-qPCR (Supplemental Figure S4A). 5-Ethynyl-2'-deoxyuridine and MTS assays showed that compared with the controls, the proliferation activities of the miR-135a-5p-overexpressing sub-cell lines were much lower $(P<0.01$ to approximately $P<0.001)$ (Figure 2, A-C). Combining with the above ISH and IHC results, the data confirm the adverse effect of miR-135a-5p on glioma cell proliferation and suggest its existence in both cell culture models and human glioma tumors.

\section{TRAF5 Is a Direct Target of miR-135a-5p in Glioma Cells}

By TargetScan version 7.0, it was found that among the target regions of miR-135a-5p in the $3^{\prime}$-UTR of the TRAF family members that were primarily concerned (TRAF1, TRAF4, TRAF5, and TRAF6), the score and ranking of TRAF5 were the highest (Supplemental Table S3). The silencing effect of the complementary pairing between miR$135 \mathrm{a}-5 \mathrm{p}$ and the $3^{\prime}$-UTR of TRAF5 mRNA was verified by dual-luciferase assays in the miR-135a-5p-overexpressing U251 and U87MG sub-cell lines (Figure 2, D and E). Moreover, the results of RT-qPCR and Western blot analysis showed that both the levels of TRAF5 mRNA and its protein were significantly lower in miR-135a$5 \mathrm{p}-$ overexpressing cell lines than in the controls $(P<0.05$ to approximately $P<0.01$ ) (Figure $2, \mathrm{~F}$ and $\mathrm{G}$ ). These results demonstrate that TRAF5 is a natural target of miR135a-5p in glioma cells. 
A
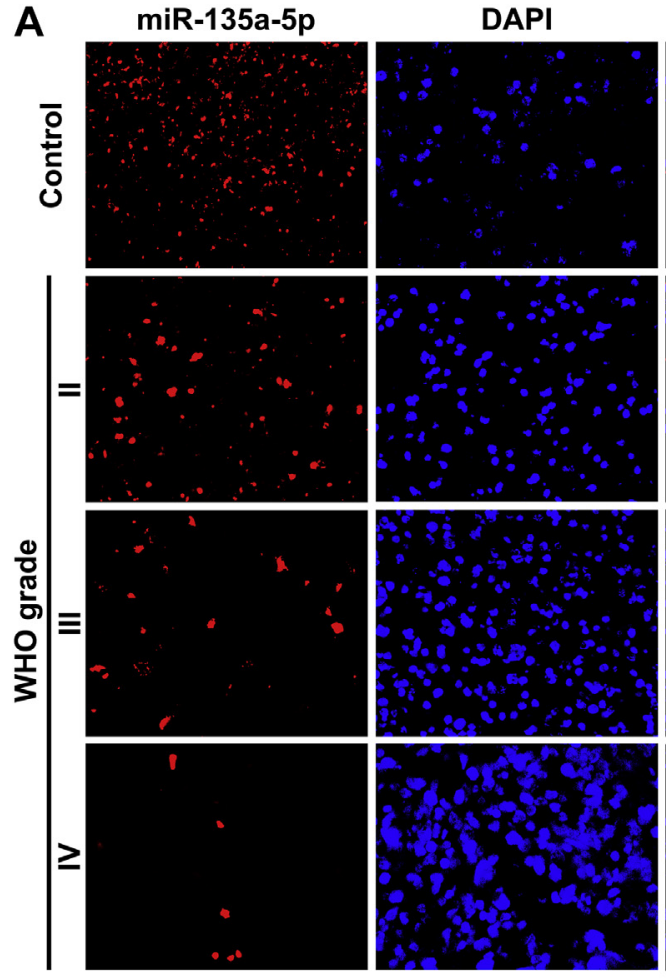

D

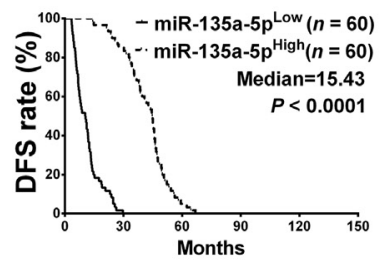

F

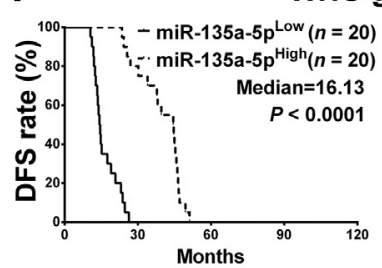

WHO grade III

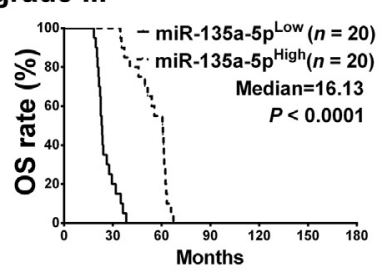

H

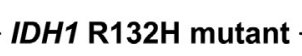
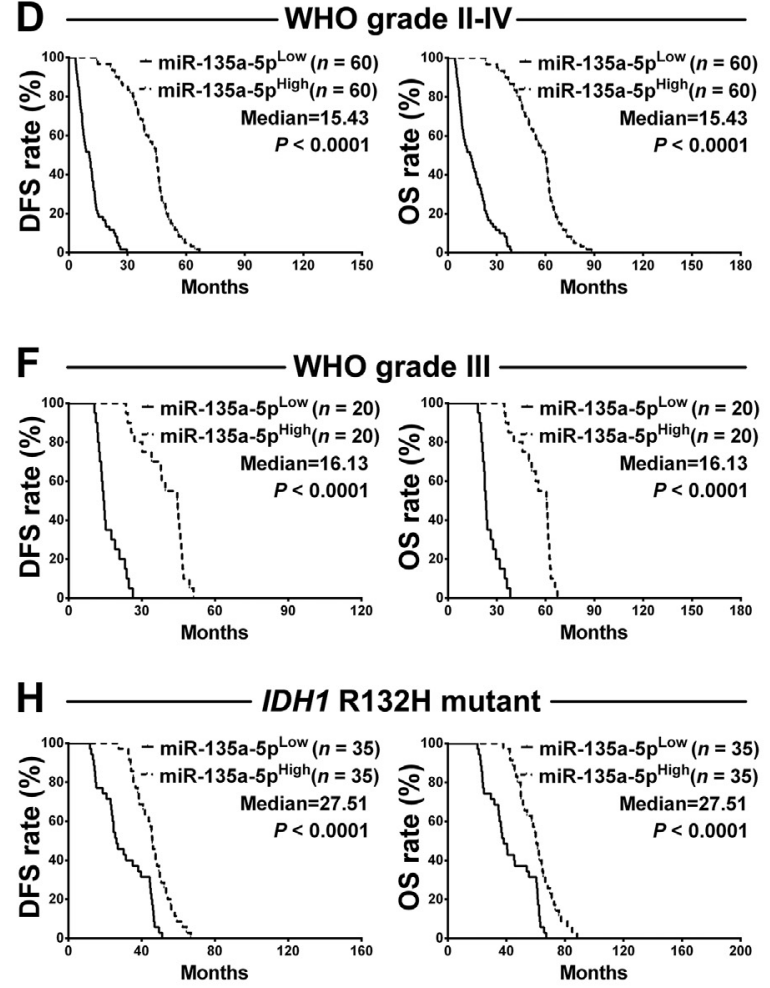

E

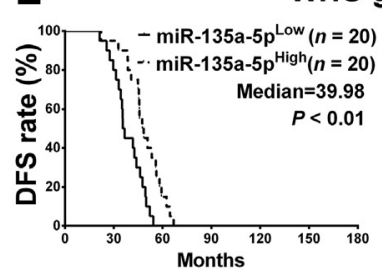

WHO grade II

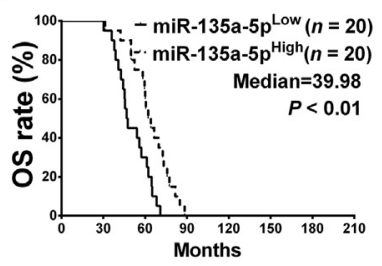

WHO grade IV
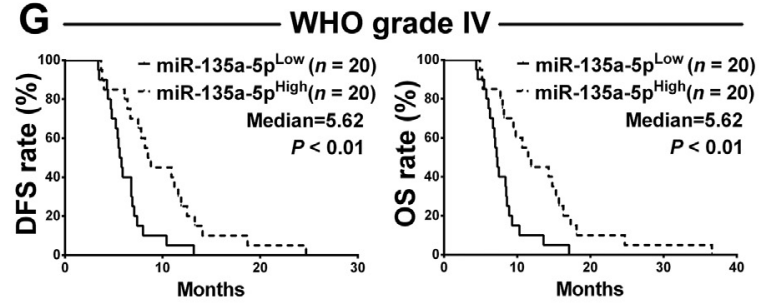

IDH1/2 wild type
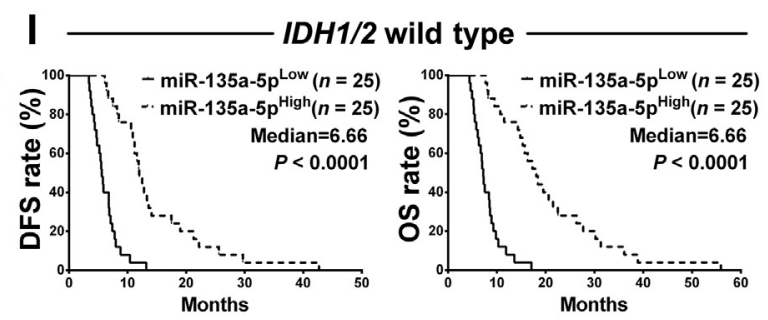

Figure 1 miR-135a-5p expression correlates with glioma grade, tumor cell proliferation, and patients' survival. A: The representative images of in situ hybridization staining of miR-135a-5p in the formalin-fixed, paraffin-embedded samples of 120 gliomas and 20 control brain tissues. B: The comparisons of the miR-135a-5p levels [labeling index (LI] among the control brain tissues and the gliomas of various grades. C: Pearson analysis reveals the inverse correlation between the LIs of miR-135a-5p and Ki-67. Both the LIs were calculated as the percentage of the positive cell number/the total cell number. D-I: Kaplan-Meier analyses of disease-free survival (DFS) and overall survival (OS). D-I: Patients were stratified into low and high expression subgroups using the median of miR-135a-5p LIs. The association between miR-135a-5p and the patients' survival is demonstrated not only in all grade glioma patients (D), but also in the patients with glioma of World Health Organization (WHO) grades II (E), III (F), and IV (G), and the patients with R132H mutant IDH1 (H) or wild-type IDH1/2 (I). Data are expressed as means \pm SD. ${ }^{* * * P}<0.001$. Scale bar $=50 \mu \mathrm{m}(\mathbf{A})$. 
miR-135a-5p and Glioma Proliferation

Table 4 Multivariate Analysis for DFS and OS in Patients with Gliomas

\begin{tabular}{|c|c|c|c|c|}
\hline \multirow[b]{2}{*}{ Factor } & \multicolumn{2}{|l|}{ DFS } & \multicolumn{2}{|l|}{ OS } \\
\hline & $\mathrm{HR}(95 \% \mathrm{CI})$ & $P$ value & $\mathrm{HR}(95 \% \mathrm{CI})$ & $P$ value \\
\hline Sex & $0.971(0.653-1.443)$ & 0.883 & $1.003(0.677-1.485)$ & 0.989 \\
\hline Predominant side & $1.099(0.786-1.536)$ & 0.580 & $1.033(0.741-1.441)$ & 0.848 \\
\hline Predominant location & $1.076(0.815-1.421)$ & 0.604 & $1.041(0.787-1.376)$ & 0.780 \\
\hline KPS & $1.001(0.976-1.028)$ & 0.914 & $1.003(0.978-1.029)$ & 0.811 \\
\hline TRAF5 LI & $1.061(1.039-1.084)$ & $<0.0001$ & $1.072(1.048-1.096)$ & $<0.0001$ \\
\hline
\end{tabular}

DFS, disease-free survival; HR, hazard ratio; KPS, Karnofsky performance score; LI, labeling index; 0S, overall survival.

\section{TRAF5 Overexpression Is Associated with Low Expression of miR-135a-5p and Poorer Prognosis}

TRAF5 immunohistochemistry was performed in the above glioma and brain specimens, and its level was found to be higher in gliomas than in brain tissues and was increased with the elevatory tumor grade $(P<0.001)$ (Figure 3, A and B). Furthermore, the TRAF5 LI correlated negatively with miR135a-5p LI $(r=-0.831, P<0.0001)$ (Figure 3C) but positively with the Ki-67 index $(r=0.946, P<0.0001)$ (Supplemental Figure S1C). Kaplan-Meier analyses found that stratifying the glioma patients with the TRAF5 levels perfectly reflected their differences in DFS $(P<0.0001)$ and OS $(P<0.0001)$ (Figure 3D). Even within the patients with the same tumor grade and $I D H$ status as well as similar age and Karnofsky performance score, higher TRAF5 LI was always associated with shorter survival (DFS, $P<0.01$ to approximately $P<0.0001$; OS, $P<0.01$ to approximately $P<0.0001$ ) (Figure 3, E-I, and Supplemental Figure S5). Meanwhile, the data from the CGGA, The Cancer Genome Atlas, and Oncomine (three data sets, including Liang et al, ${ }^{23}$ Bredel et al, ${ }^{24}$ and The Cancer Genome Atlas) all showed that the TRAF5 mRNA level was significantly higher in glioblastoma and predicted poorer prognosis (Supplemental Figure S3, B-D). Cox regression verified that TRAF5 LI was another independent survival predictor in parallel with miR135a-5p LI and IDH status (Table 4 and Supplemental Table S2). These results also manifested that miR-135a-5p shortage was an important cause for the excessive expression of TRAF5 in glioma cells.

\section{miR-135a-5p Blocks the Proliferation of Glioma Cells by Silencing TRAF5}

To further interpret the inhibitory effect of miR-135a-5p on glioma cell proliferation, the cell cycle distribution of the miR-135a-5p-overexpressing sub-cell lines and their controls was compared, and the percentages of the $\mathrm{G}_{0} / \mathrm{G}_{1}$ cells were found to be significantly higher in the former $(P<0.01)$ (Figure 4, A and B). The miR-135a-5p-overexpressing sub-cell lines were then infected with TRAF5 lentivirus, which had been proved to highly efficiently express TRAF5 in glioblastoma cell lines (Supplemental Figure S4, B-D). Flow cytometry assay results showed TRAF5 lentivirus infection completely abrogated the blocking effects of miR-135a-5p on $\mathrm{G}_{1} / \mathrm{S}$-phase transition $(P<0.01)$ (Figure $4, \mathrm{~A}$ and $\mathrm{B}$ ). Consistently, the miR-135a-5p-overexpressing sub-cell lines showed lower proliferation ability than their controls, whereas the proliferation activities of the TRAF5 lentivirus-infected sub-cell lines were restored almost to the control levels $(P<0.01$ to approximately $P<0.001)$ (Figure 4, C-E). In addition, knocking down the endogenous TRAF5 with the specific siRNAs (siTRAF5\#1 and siTRAF5\#2) perfectly imitated the above effects of miR135a-5p $(P<0.05$ to approximately $P<0.001)$ (Figure 5, and Supplemental Figure S6). The above results of proliferation experiments were completely repeated in the U87MG sub-cell lines maintained in nonserum neurosphere medium $(P<0.01$ to approximately $P<0.001$ ) (Supplemental Figure S7). In nude mice experiments, the xenograft tumors overexpressing miR-135a-5p formed by U87MG cells (Figure 6) and primary GBM cells (Figure 7) showed lower growth rate, cell density, and TRAF5 and Ki-67 levels compared with the controls, whereas the host mice enjoyed less weight loss and longer survival $(P<0.05$ to approximately $P<0.001$ ) (Figures 6 and 7). However, these tumor suppressive effects were largely compromised by TRAF5 overexpression $(P<0.05$ to approximately $P<0.001)$ (Figures 6 and 7). All these results indicate that miR-135a-5p prevents glioma cells from $\mathrm{G}_{1} / \mathrm{S}$-phase transition and proliferation by targeting TRAF5 both in vitro and in vivo.

miR-135a-5p Restrains Glioma Cell Proliferation by the TRAF5-AKT Pathway

To explore the molecular pathway through which miR135a-5p restrains glioma cell proliferation, AKT, c-Myc, and cyclin D1, the crucial effectors downstream of TRAF5, were studied. TRAF5 down-regulation significantly reduced the levels of phosphorylated AKT, c-Myc, and cyclin D1 $(P<0.01$ to approximately $P<0.001)$ (Figure $8, A-D)$ in the miR-135a-5p overexpressing and TRAF5-silenced U251 
A
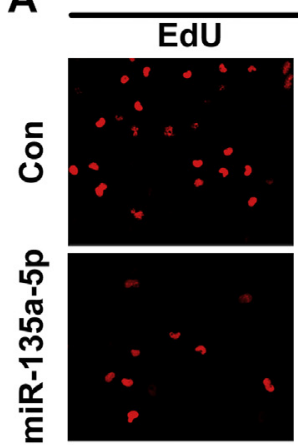

B

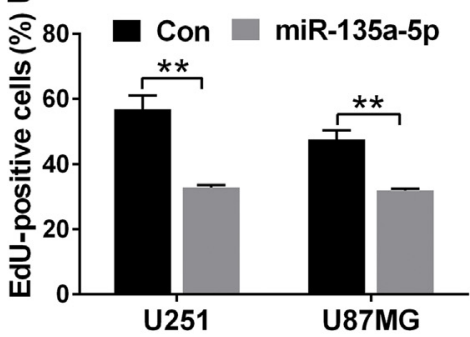

U251

D Target region

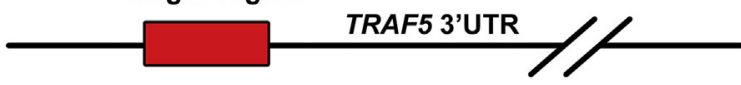

has-miR-135a-5p

3' aguguauccuuauuU Uucgguau 5'

Position 422 to 428 of p-WT

5'...G CCUUAAGUCUGCAAAAG C CAUUC...3'

Fragment of p-MT

5'...GCCUUAAGUCUGCAAAAGEGAUC...3'
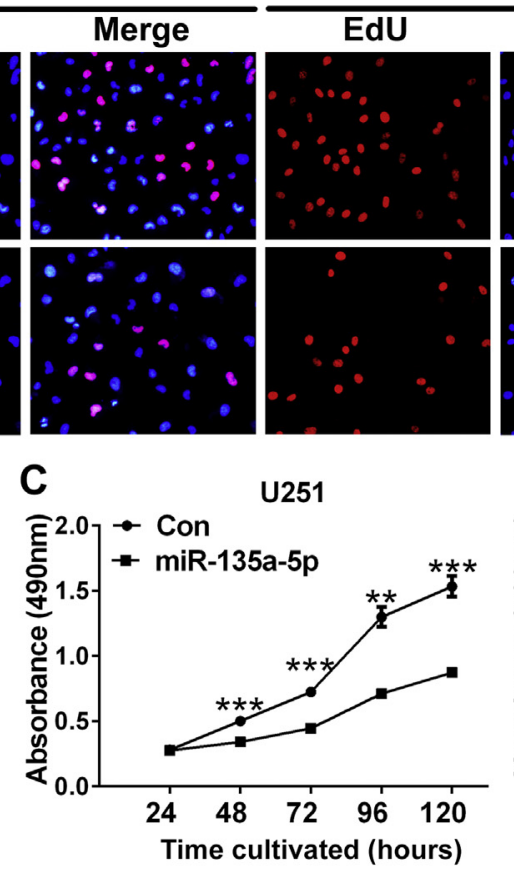

U87MG
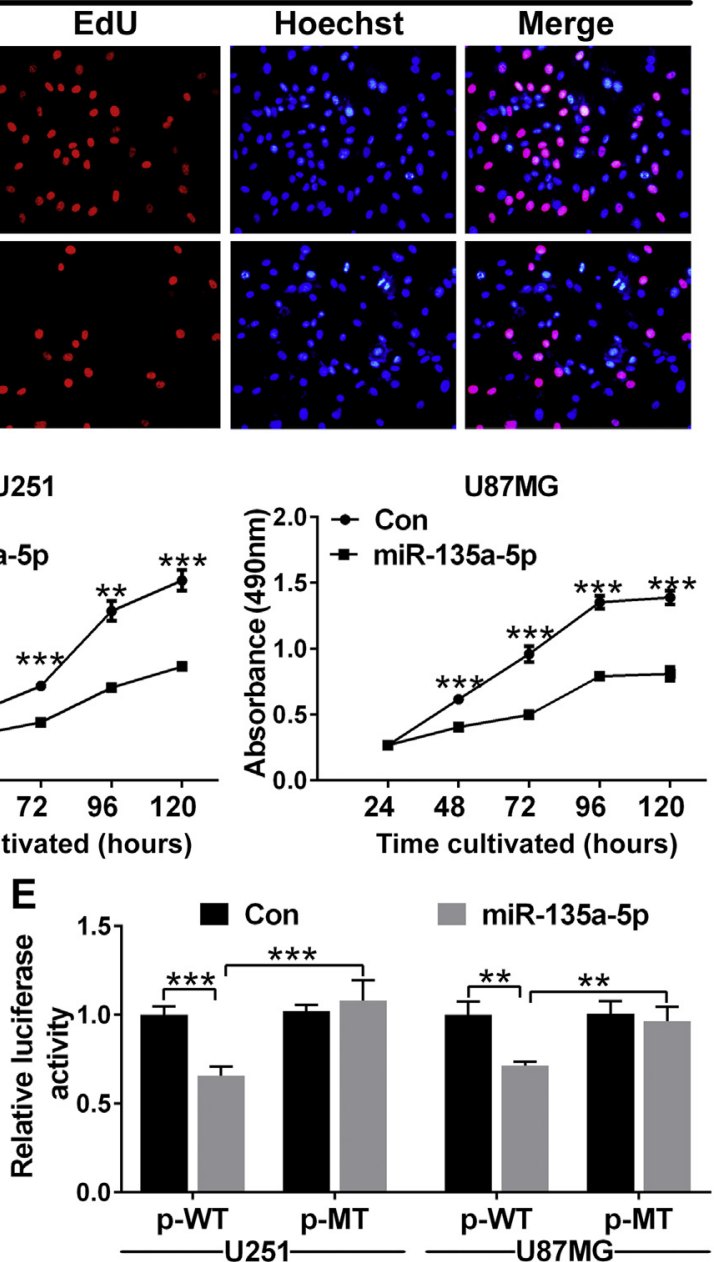

F
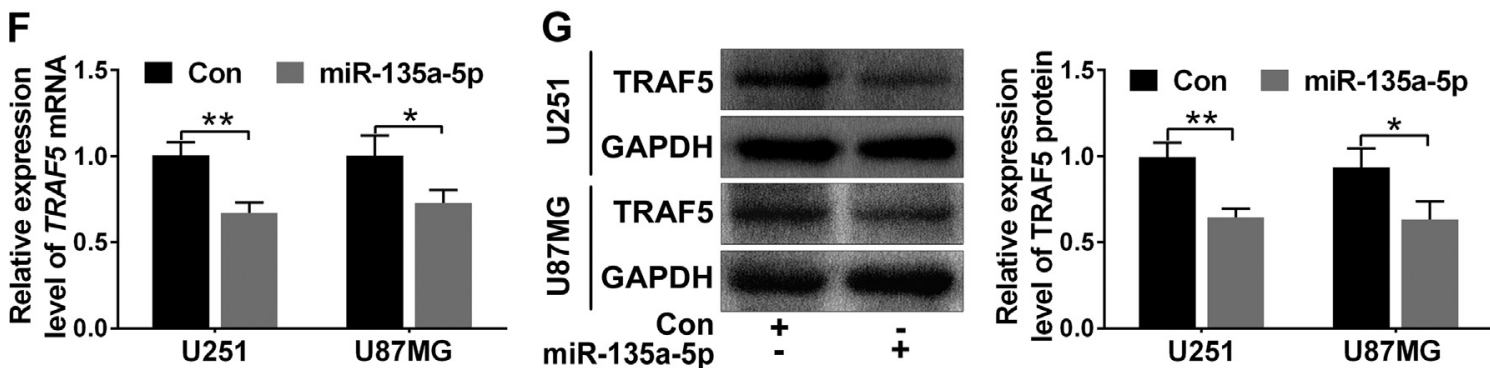

Figure 2 miR-135a-5p functions as a glioma suppressor by directly targeting TRAF5. A: The 5-ethynyl-2'-deoxyuridine (EdU) assay of the control (Con) and the miR-135a-5p-overexpressing U251 and U87MG sub-cell lines. B: The comparisons of the proliferating activities between the sub-cell lines, as indicated. The EdU-positive and Hoechst-stained cells were counted under microscopic fields. C: The growth curves of the indicated sub-cell lines obtained from the 3-(4, 5-dimethylthiazol-2-yl)-5-(3-carboxymethoxyphenyl)-2-(4-sulfophenyl)-2H-tetrazolium (MTS) assays. D: miR-135a-5p binds to the 3'-untranslated region (UTR) of TRAF5 mRNA by complementary base paring. The blue boxed area indicates the seed sequence of miR-135a-5p and its target region in the $3^{\prime}-$ UTR of TRAF5 mRNA. The target region also exists in the transcript of the wild-type reporter plasmid TRAF5-3'-UTR-wild type (WT; $p$-WT) but is absent from the transcript of the mutant (MT) reporter plasmid TRAF5-3'-UTR-MT (p-MT). E: The dual-luciferase reporter assay results of the control and the miR-135a$5 \mathrm{p}$-overexpressing sub-cell lines transfected with the reporter plasmid, as indicated. $\mathbf{F}$ and $\mathbf{G}$ : Real-time quantitative RT-PCR and Western blot detection of TRAF5 mRNA and protein in the cells, as indicated. TRAF5 mRNA and its protein levels were normalized by those of glyceraldehyde-3-phosphate dehydrogenase $(\mathrm{GAPDH})$, with the results of the control groups artificially set to 1.0. All experiments were performed at least in triplicate. Data are expressed as means \pm SD. ${ }^{*} P<0.05,{ }^{*} P<<0.01$, and ${ }^{* * *} P<0.001$. Original magnification, $\times 200(\mathrm{~A})$.

and U87MG cells, but the total AKT levels remained unchanged $(P>0.05)$ (Figure 8, A-D). The changes in miR$135 \mathrm{a}-5 \mathrm{p}$-overexpressing cell lines could be effectively reversed by TRAF5 overexpression $(P<0.01$ to approximately $P<0.001$ ) (Figure $8, \mathrm{~A}$ and B). These results demonstrate that miR-135a-5p inhibits the activation of the TRAF5-AKT pathway by directly targeting TRAF5, and it thereby decreases the expressions of c-Myc and cyclin D1. 
A

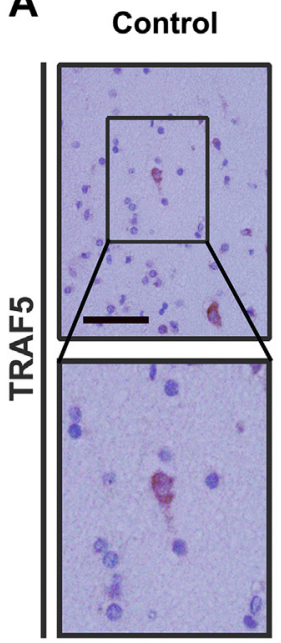

Nontumoral brain tissue

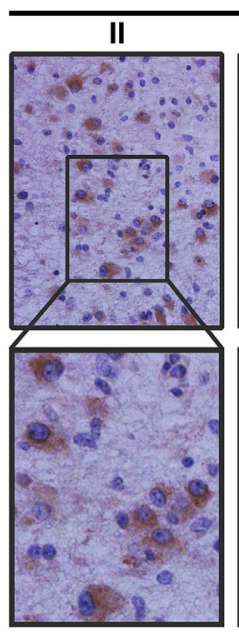

Diffuse astrocytoma
WHO grade

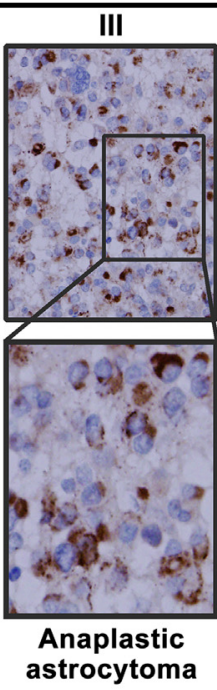

WHO grade II-IV
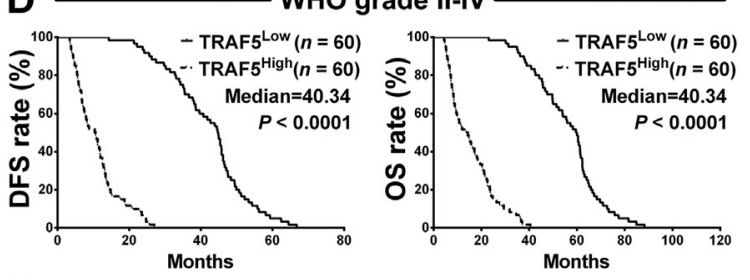

$\mathbf{F}$
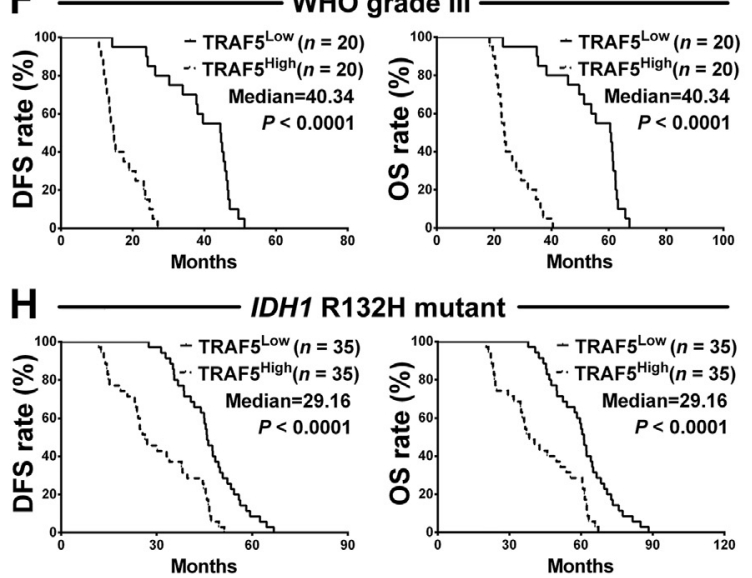

B
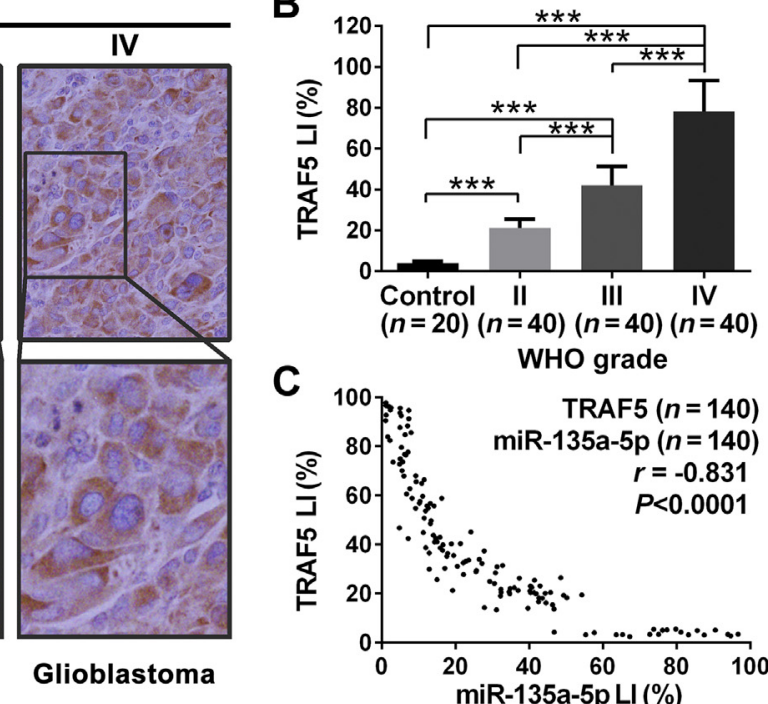

C

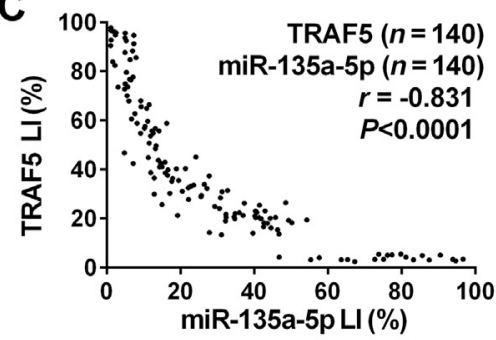

WHO grade II
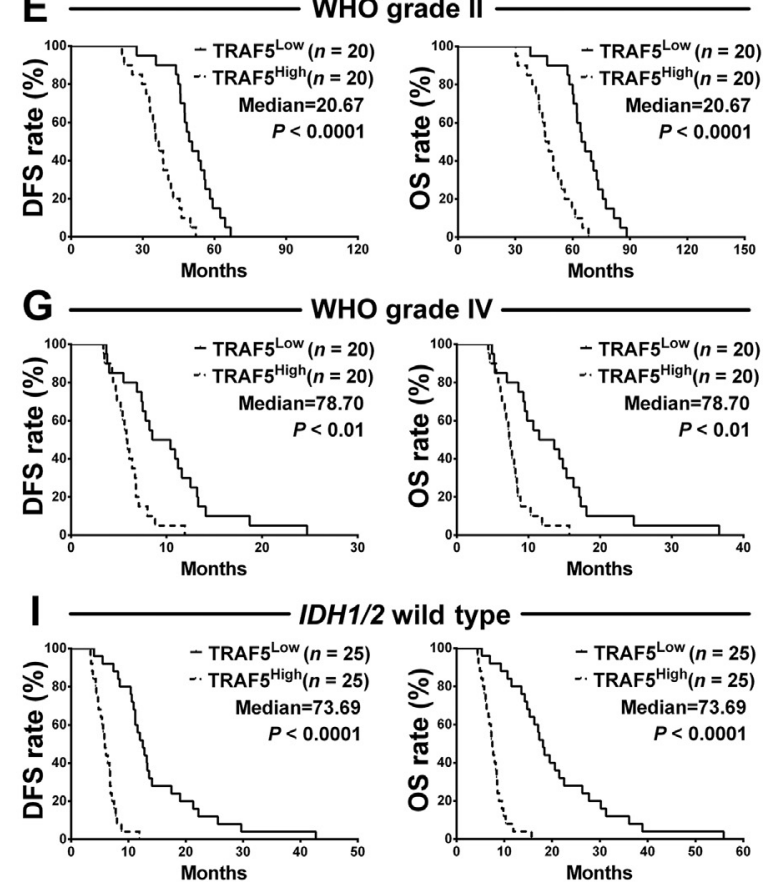

Figure 3 TRAF5 expression correlates with glioma grade, miR-135a-5p expression, and the patients' survival. A: The representative images of TRAF5 immunohistochemistry. Boxed areas in the top row are shown in higher magnification in the bottom row. B: The comparisons of the TRAF5 levels [labeling index (LI] among the control brain tissues and the gliomas of various grades. The TRAF5 LI was calculated as those of miR-135a-5p and Ki-67. C: Pearson analysis demonstrates the inverse correlation between the expression levels of TRAF5 and miR-135a-5p. D-I: Kaplan-Meier analyses of disease-free survival (DFS) and overall survival (OS). Patients were stratified into low and high expression subgroups using the median of TRAF5 LIs. D-I: The association between TRAF5 and the patients' survival is demonstrated not only in all grade glioma patients (D), but also in the patients with glioma of World Health Organization (WHO) grades II (E), III (F), and IV (G), and the patients with R132H mutant IDH1 (H) or wild-type IDH1/2 (I). Data are expressed as means \pm SD. $* * * P<0.001$. Scale bar $=50 \mu \mathrm{m}(\mathbf{A})$. Original magnification, $\times 400$ (A, top row).

Through this pathway, miR-135a-5p exerts its inhibitory effects on the $\mathrm{G}_{1} / \mathrm{S}$-phase transition and proliferation of glioma cells (Figure 8E).

\section{Discussion}

Although miR-135a-5p has been reported as a tumor suppressor in several human neoplasms, ${ }^{13-16}$ its clinical relevance and exact roles in gliomas remain largely unknown. In the present study, the glioma suppressive effects of miR-135a-5p have been demonstrated by investigating its antiproliferative capacity both in vitro and in vivo; for the first time, TRAF5 was identified as its functional target. Most important, it was confirmed that both the miR-135a-5p and TRAF5 levels were independent predictors for glioma patient survival, which highlights their practical values as prognostic biomarkers. 
A

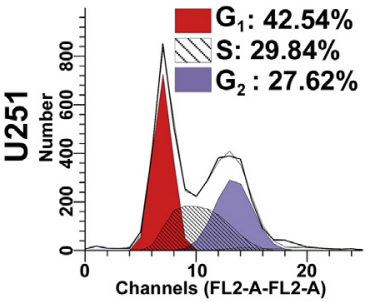

Con

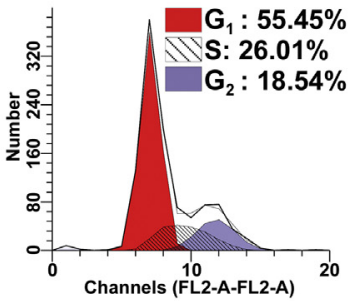

miR-135a-5p

B

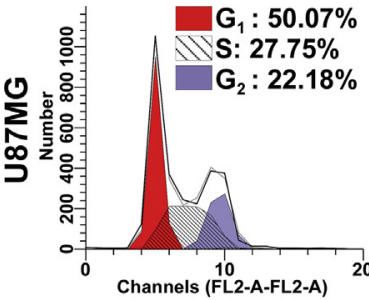

Con

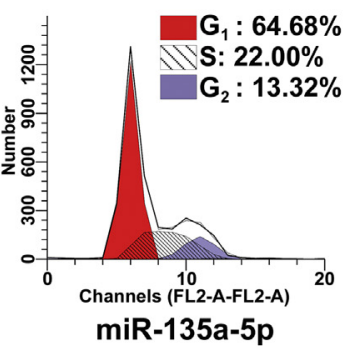

miR-135a-5p

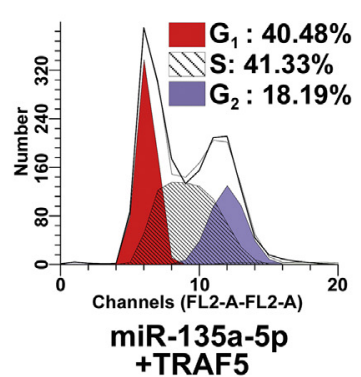

+TRAF5

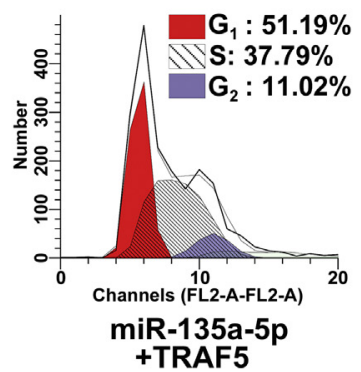

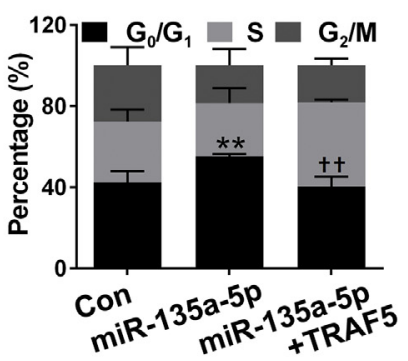

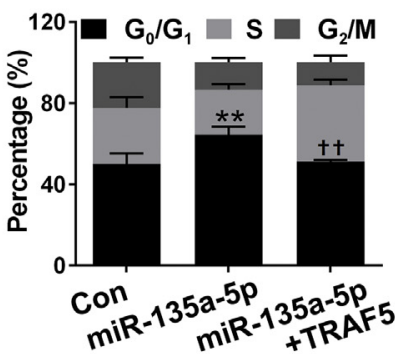

C

U251

U87MG
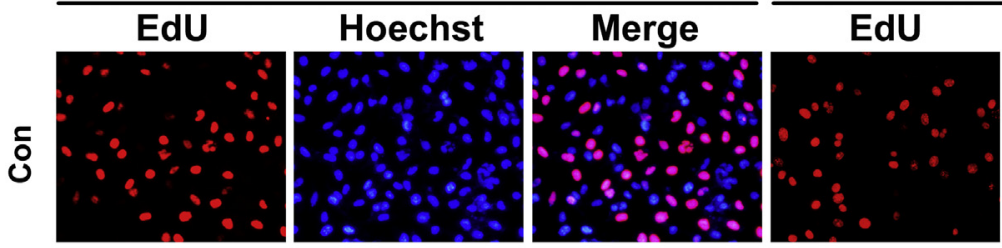

\section{Hoechst}
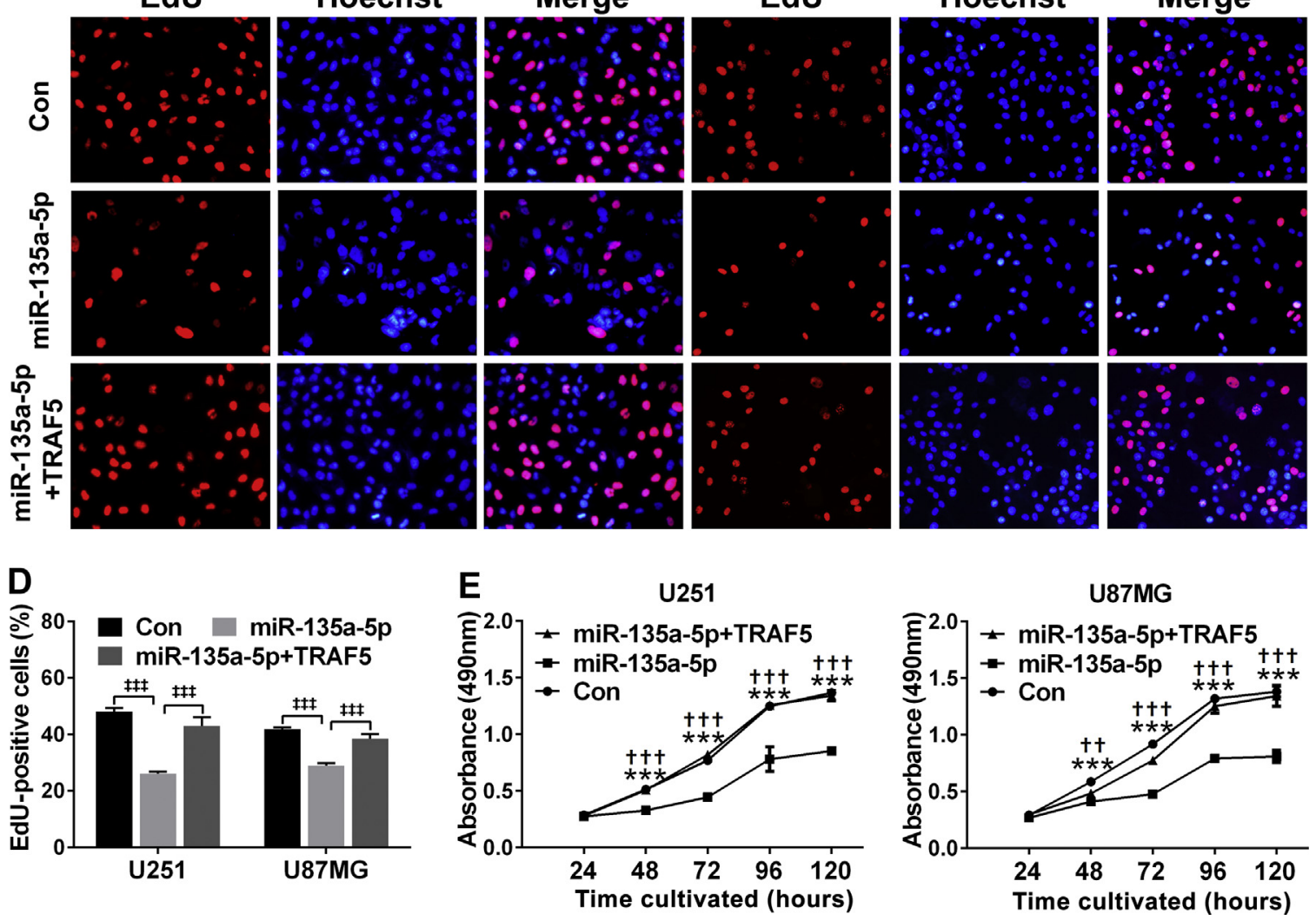

Figure 4 TRAF5 is the functional target by which miR-135a-5p restrains cell cycle progression and glioma cell proliferation. A and B: The representative flow cytometry assay cell cycle analysis results (left panels) and the percentages of each phase (right panel). U251 and U87MG cells of the control (Con), the miR-135a-5p, and the miR-135a-5p+TRAF5 groups were infected with the control lentivirus, the miR-135a-5p-expressing lentivirus, and the miR-135a$5 p+$ TRAF5-expressing lentivirus, respectively. FL2-A represents the total fluorescence intensity measured by the channel detecting propidium iodide-stained DNA of cell population. C and D: The 5-ethynyl-2'-deoxyuridine (EdU) assay results of proliferating activities and their comparisons in the above groups. E: The growth curves of the above groups obtained from the 3-(4, 5-dimethylthiazol-2-yl)-5-(3-carboxymethoxyphenyl)-2-(4-sulfophenyl)-2H-tetrazolium (MTS) assays. All the experiments were performed at least in triplicate. Data are expressed as means \pm SD. ${ }^{* *} P<0.01,{ }^{* * *} P<0.001$, compared with the Con group; ${ }^{\dagger \dagger} P<0.01,{ }^{\dagger \dagger} P<0.001$, compared with the miR-135a-5p group; ${ }^{\ddagger \ddagger} P<0.001$. Original magnification, $\times 200$ (C). 


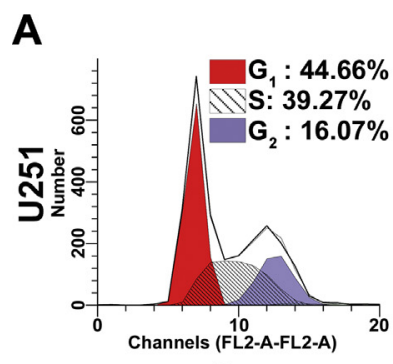

Scr

B

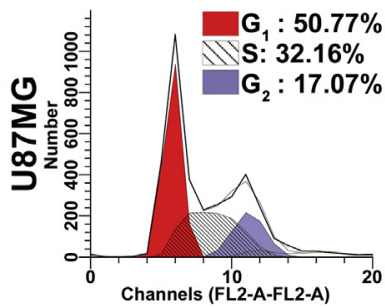

Scr

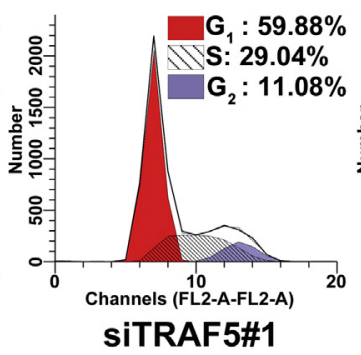

SITRAF5\#1

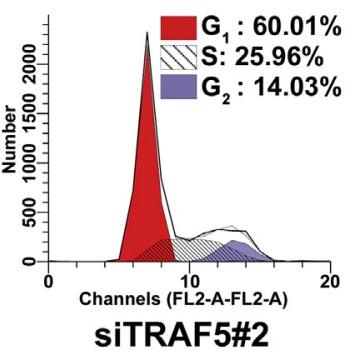

SITRAF5\#2

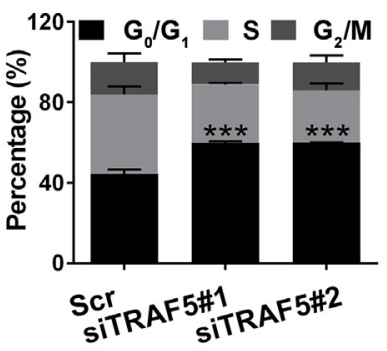

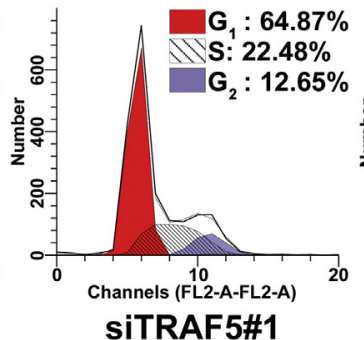

SITRAF5\#1
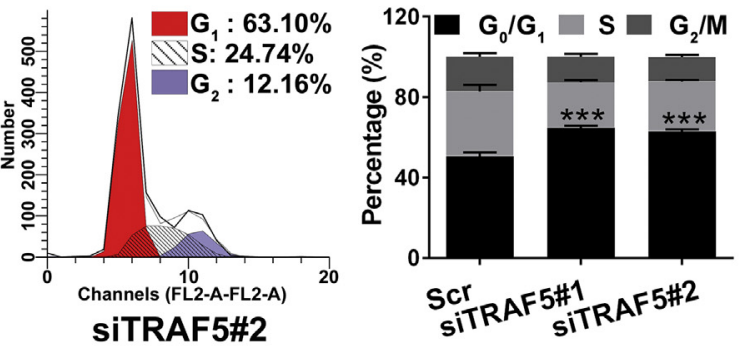

C

U251

U87MG
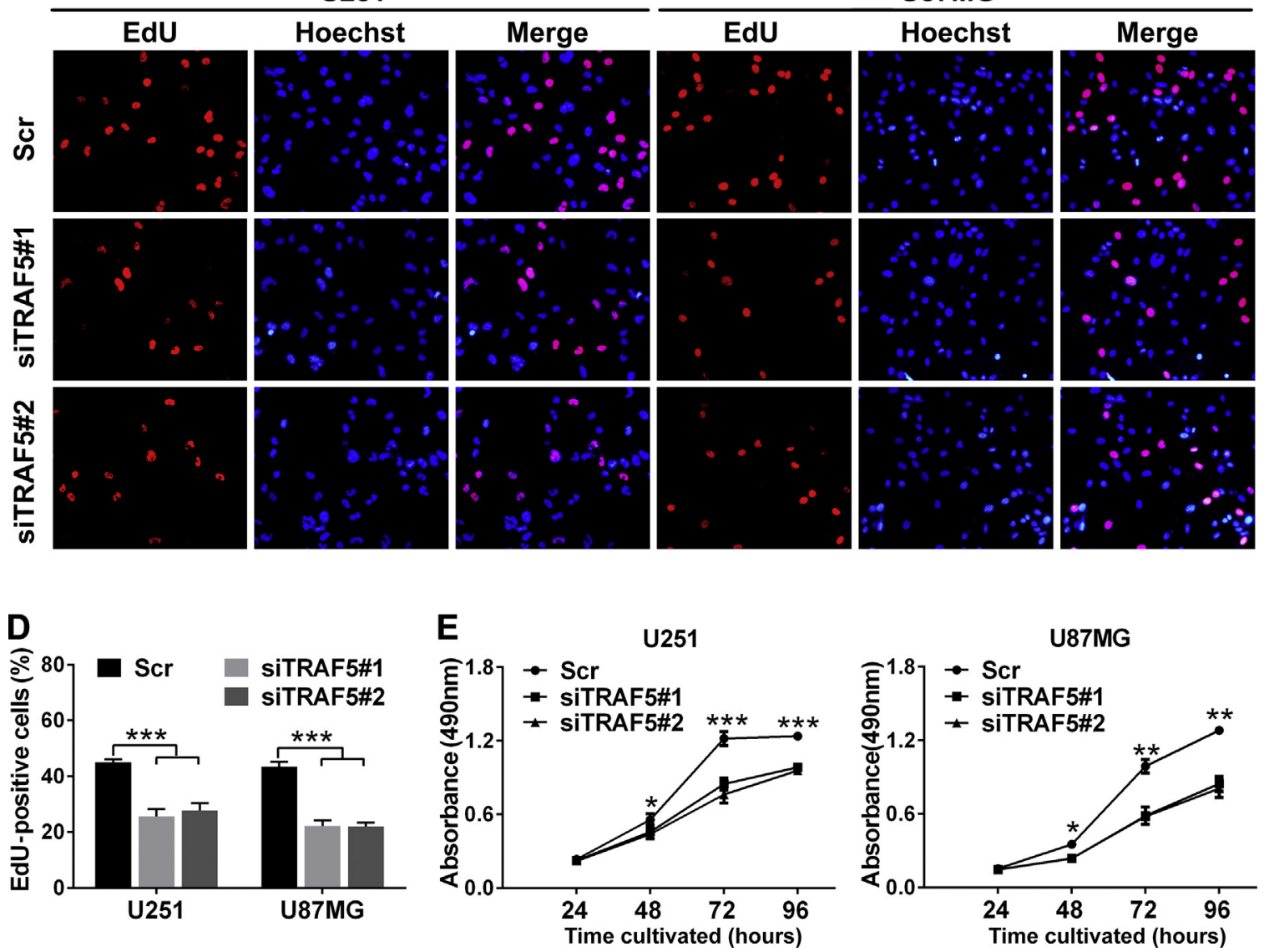

Figure 5 Silencing the endogenous TRAF5 suppresses the proliferation of glioma cells. A and B: The representative flow cytometry assay cell cycle analysis results (left panels) of the U251 and U87MG cells transfected with either the scrambled control sequence (Scr) or the TRAF5 siRNAs (siTRAF5\#1 and siTRAF5\#2), and percentages of each phase (right panel). FL2-A represents the total fluorescence intensity measured by the channel detecting propidium iodide-stained DNA of cell population. $\mathbf{C}$ and D: The 5-ethynyl-2'-deoxyuridine (EdU) assay results of proliferating activities and their comparisons in the above groups. E: The growth curves of the above cells obtained from the 3-(4, 5-dimethylthiazol-2-yl)-5-(3-carboxymethoxyphenyl)-2-(4-sulfophenyl)-2Htetrazolium (MTS) assays. All the experiments were performed at least in triplicate. Data are expressed as means \pm SD. ${ }^{*} P<0.05,{ }^{*} P<0.01$, and $* * * P<0.001$. Original magnification, $\times 200$ (C). 
A

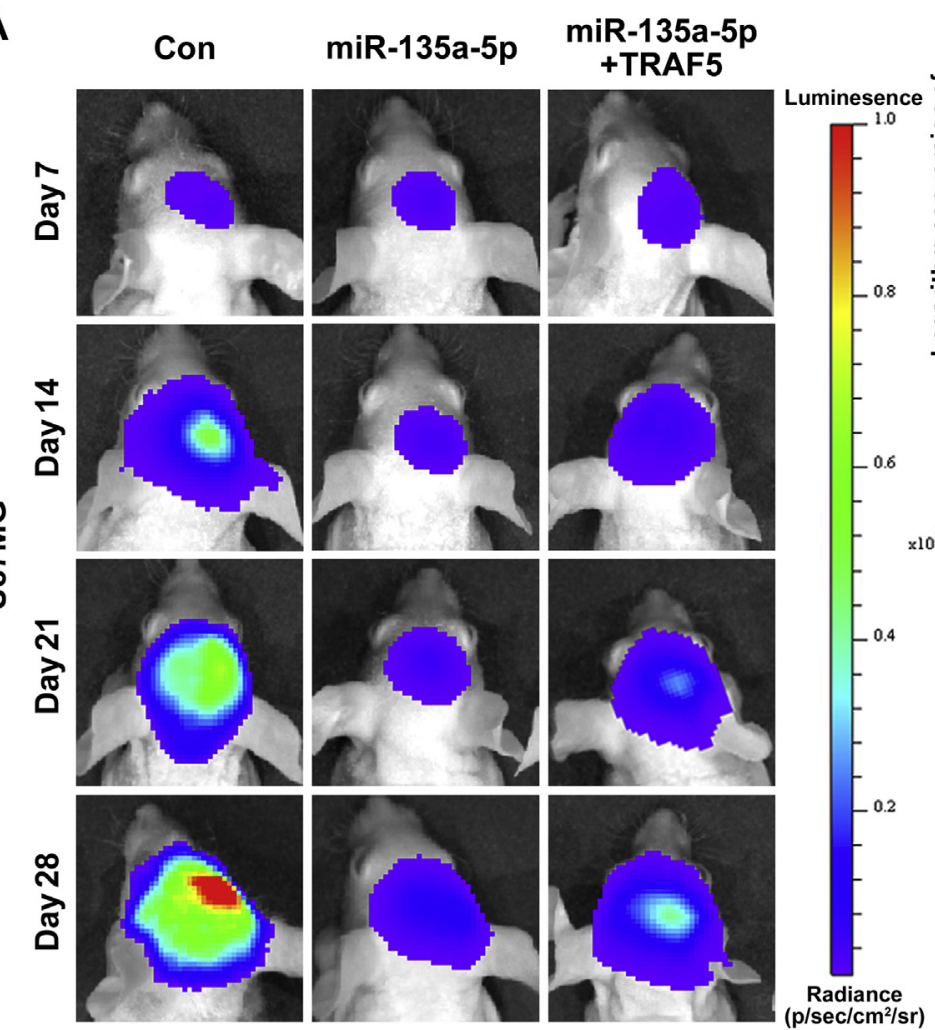

$\mathbf{E}$
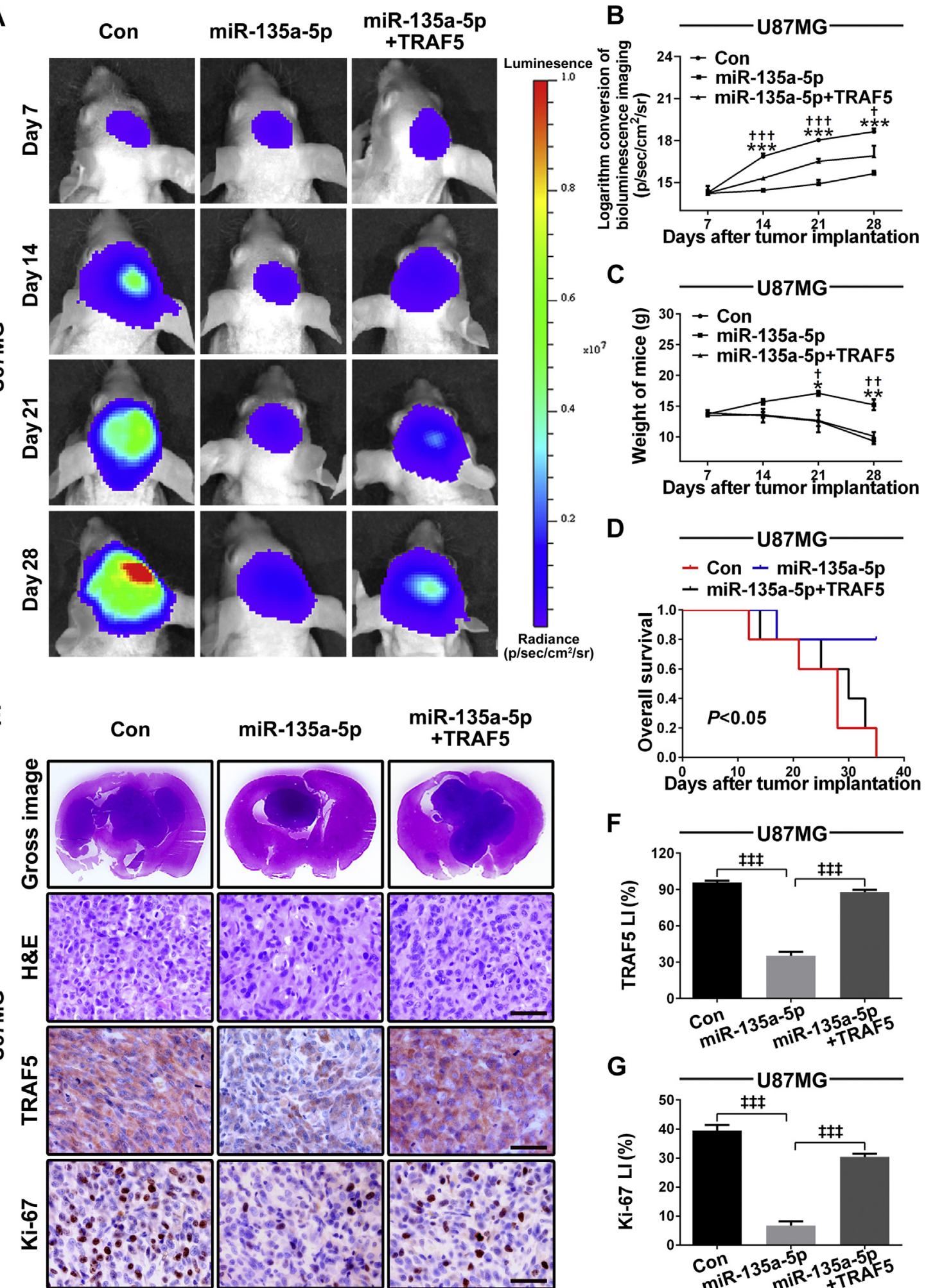

C

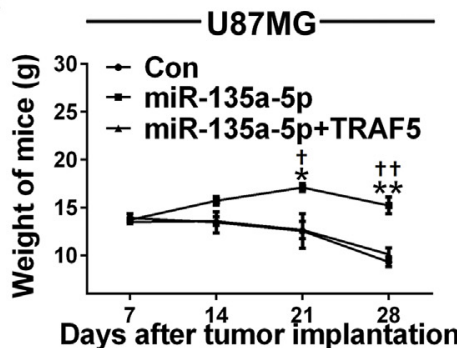

D

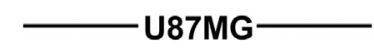

- Con - miR-135a-5p

- miR-135a-5p+TRAF5

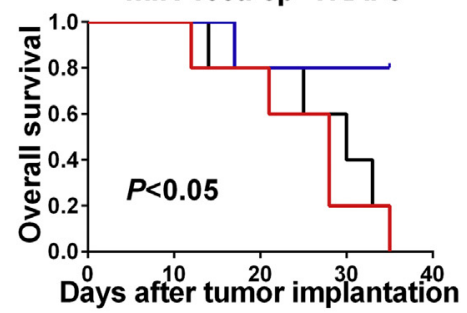

$\mathbf{F}$

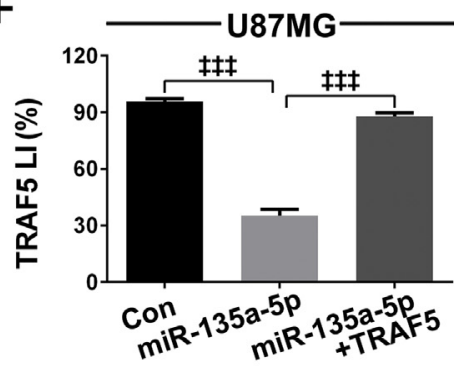

G

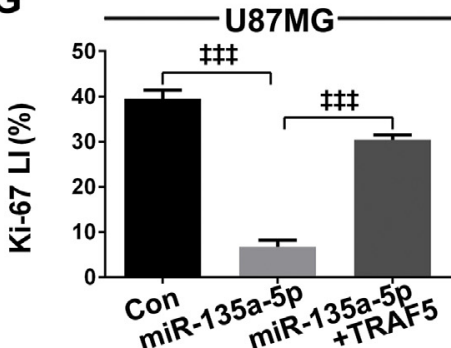

Figure 6 miR-135a-5p impedes xenograft tumor growth in tumor-bearing mice and prolongs their survival by targeting TRAF5. A and B: The bioluminescent images (A) and the corresponding quantification (B) of the xenograft tumors in the mice implanted with the control (Con), the miR-135a$5 p$-overexpressing (miR-135a-5p) U87MG cells, or the miR-135a-5p+TRAF5-overexpressing (miR-135a-5p+TRAF5) U87MG cells. C and D: The curves of the weight (C) and survival (D) of the tumor-bearing mice, as indicated. E: Hematoxylin and eosin (H\&E) staining (first and second rows) and immunohistochemical staining (third and fourth rows) of the indicated xenograft tumors. $\mathbf{F}$ and $\mathbf{G}$ : The comparisons of the labeling indexes (LIs) of TRAF5 (F) and Ki-67 (G) in the above xenograft tumor tissues. All experiments were performed at least in quintuplicate. Data are expressed as means \pm SD. ${ }^{*} P<0.05$, ${ }^{*} P<0.01$, and ${ }^{* * *} P<0.001$ compared with the Con group; ${ }^{\dagger} P<0.05$, ${ }^{\dagger \dagger} P<0.01$, and ${ }^{\dagger \dagger \dagger} P<0.001$ compared with the miR-135a-5p group; ${ }^{\ddagger \pm \ddagger} P<0.001$. Scale bar $=50 \mu \mathrm{m}(\mathbf{E})$. 
A

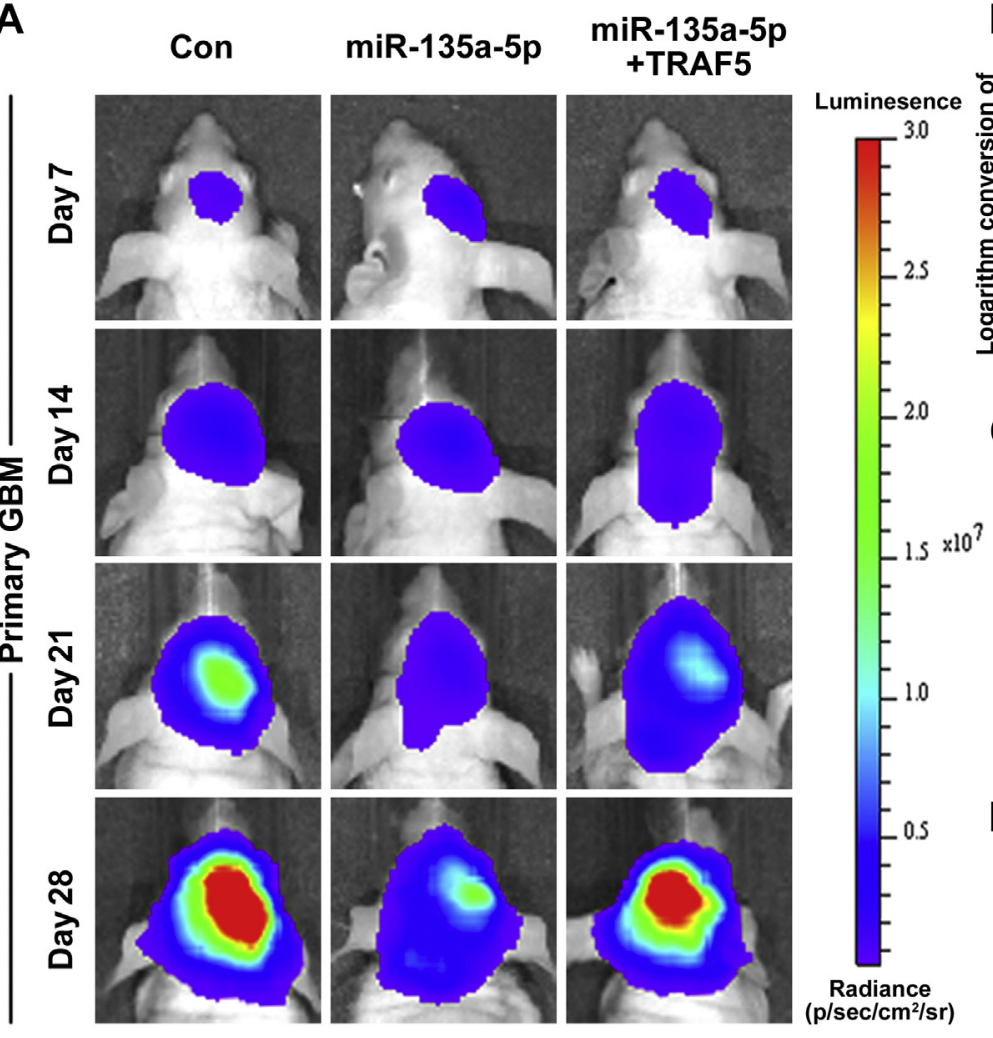

E

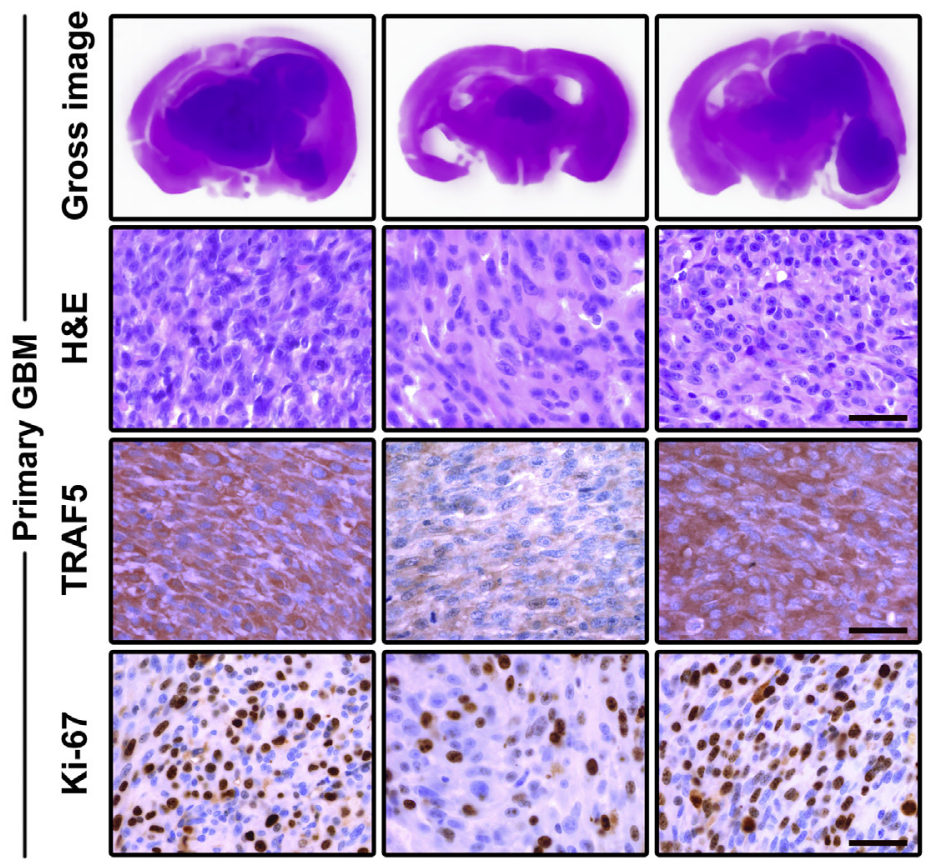

B

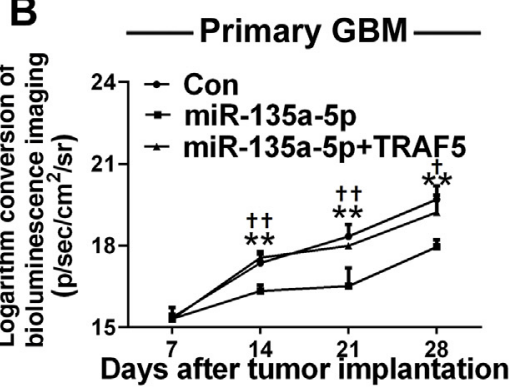

C

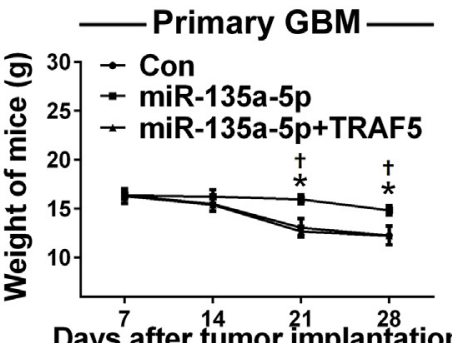

Days after tumor implantation

D
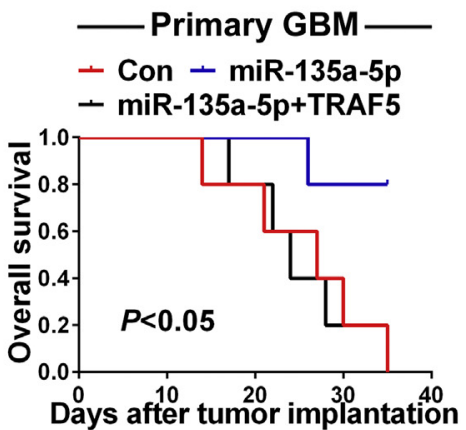

$\mathbf{F}$

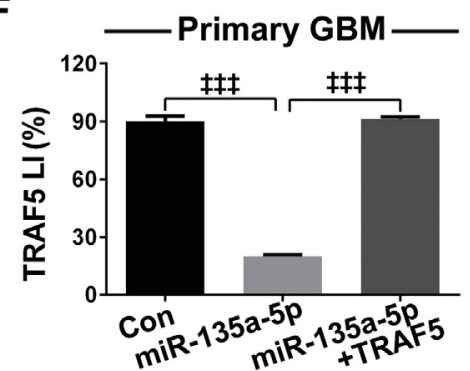

G

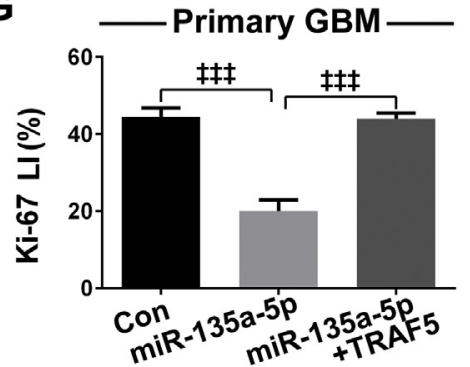

Figure 7 miR-135a-5p exerts antitumoral effects in xenografts of the primary glioblastoma cells. $\mathbf{A}$ and $\mathbf{B}$ : The bioluminescent images (A) and the corresponding quantification (B) of patient-derived xenograft models [primary glioblastoma (GBM)] in the groups of control (Con), miR-135a$5 p$-overexpressing (miR-135a-5p) cells, or miR-135a-5p+TRAF5-overexpressing (miR-135a-5p+TRAF5) cells. C and D: The curves of the weight (C) and survival (D) of the tumor-bearing mice, as indicated. E: Hematoxylin and eosin (H\&E) staining (first and second rows) and immunohistochemical staining (third and fourth rows) of the indicated xenograft tumors. $\mathbf{F}$ and $\mathbf{G}$ : The comparisons of the labeling indexes (LIs) of TRAF5 (F) and Ki-67 (G) in the above xenograft tumor tissues. All experiments were performed at least in quintuplicate. Data are expressed as means \pm SD. ${ }^{*} P<0.05,{ }^{* *} P<0.01$ compared with the Con group; ${ }^{\dagger} P<0.05,{ }^{\dagger \dagger} P<0.01$ compared with the miR-135a-5p group; ${ }^{\ddagger \ddagger} P<0.001$. Scale bar $=50 \mu \mathrm{m}(\mathbf{E})$. 

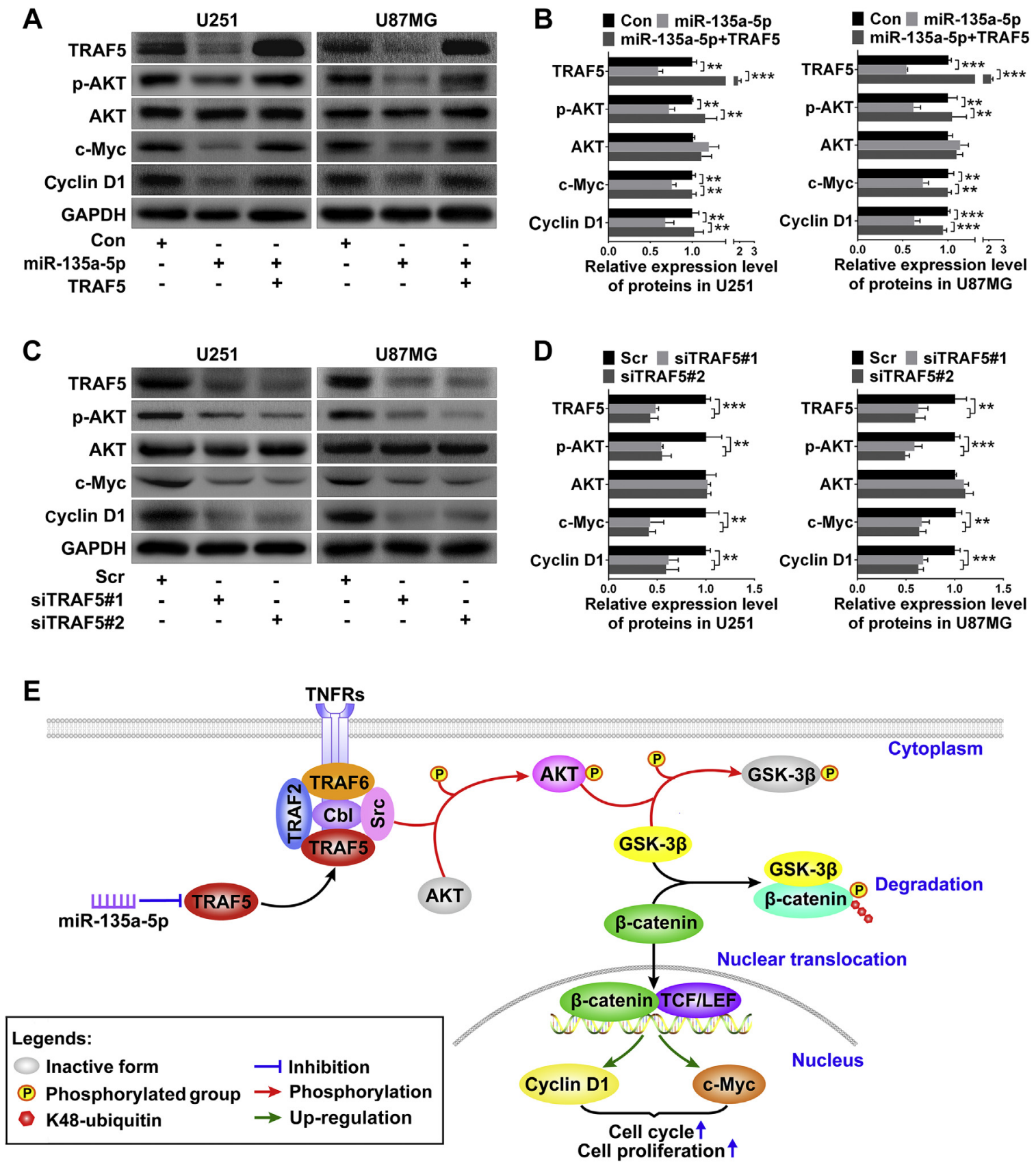

Figure 8 miR-135a-5p suppresses the phosphorylation of AKT and the expression of cyclin D1 and c-Myc. A and B: The Western blot analyses of TRAF5, phosphorylated AKT (p-AKT), AKT, c-Myc, and cyclin D1 in the U251 and U87MG cells of the control (Con), miR-135a-5p, and miR-135a-5p+TRAF5 groups (A) and the comparison among groups of their expressions (B). C and D: The Western blot analyses of the above proteins in the U251 and U87MG cells transfected by either scrambled control sequence ( $\mathrm{Scr}$ ) or TRAF5 siRNAs (siTRAF5\#1 and siTRAF5\#2); these protein levels were compared. All the protein levels were normalized by that of glyceraldehyde-3-phosphate dehydrogenase (GAPDH). All the experiments were performed at least in triplicate. Data are expressed as means \pm SD. E: Schematic illustration of the molecular pathway by which miR-135a-5p induces $G_{1}$ phase arrest and inhibits glioma cell proliferation. ${ }^{*} P<0.01,{ }^{* * *} P<0.001$. GSK-3 $\beta$, glycogen synthase kinase; LEF, lymphoid enhancing factor; TCF, T-cell factor; TNFRs, tumor necrosis factor receptor superfamily.

These results prove that several miRNAs may help in the diagnosis and prognosis assessment of gliomas. ${ }^{6,7,21,22,25}$ These findings provide useful clues and solid evidence for biomarker screening. Herein, it was confirmed that the expression of miR-135a-5p or TRAF5 decreased or increased, respectively, with the grade increase in the 120 human glioma specimens tested. Similarly, the data from the
CGGA, The Cancer Genome Atlas, and Oncomine also demonstrated the differences of their expressions among World Health Organization grade II, III, and IV gliomas, implying their potential usefulness in glioma grading. Moreover, the associations of these molecules with the patients' survival were confirmed not only in all grade gliomas, but also in gliomas with the same grade, gliomas with 
the same $I D H$ status, and patients with similar ages and Karnofsky performance scores. Therefore, taking their expression levels for consideration could substantially improve the accuracy of grading and the prognostic prediction of gliomas.

Glioblastomas, accounting for $60 \%$ to $70 \%$ of malignant gliomas, obtain the abilities of speedy growth and aggressiveness at least partly because of the excessive proliferation of the tumor cells. ${ }^{3,26}$ The antiproliferative effect of miR$135 a-5 p$ was initially proposed by the inverse correlation between its LI and that of Ki-67 in glioma specimens and then verified by the in vitro and in vivo studies with the miR-135a-5p-overexpressing glioblastoma sub-cell lines. Cell cycle analysis also showed that miR-135a-5p blocked the $G_{1} / S$-phase transition and increased the $G_{1}$ phase percentage of glioblastoma cells. These results manifest that the abnormal decrease of miR-135a-5p is an important cause leading to the uncontrolled proliferation of glioma cells, especially in high-grade gliomas.

To explain how miR-135a-5p suppresses glioma cell proliferation, the TRAF family, the members of which were the important regulators of AKT, was studied. ${ }^{27-29}$ Bioinformatic prediction shows that TRAF1, TRAF4, TRAF5, and TRAF6 are all of the potential targets of miR-135a-5p, and the comprehensive score of TRAF5 is the highest. TRAF5 was identified as a functional target of miR-135a-5p by bioinformatics prediction, luciferase reporter assay, and direct observation of the silencing effect in glioblastoma cell lines. As a key cofactor of TRAF6, TRAF5 can be recruited by the activated tumor necrosis factor receptor superfamily to assemble the TRAF5-TRAF2-TRAF6-Cbl complex. The complex will then recruit Src kinase to induce the phosphorylation and activation of AKT. ${ }^{27,30}$ These data show that the phosphorylated AKT levels in the miR-135a$5 \mathrm{p}-$ overexpressing glioma sub-cell lines are significantly lower and may be completely rescued by exogenous TRAF5, indicating that TRAF5 is the pivot factor mediating the inhibitory effect of miR-135a-5p on the AKT pathway.

Cyclin D1 and c-Myc are the key drivers of $\mathrm{G}_{1} / \mathrm{S}$-phase transition, and their expressions are strictly controlled by $\beta$-catenin and the transcription factor T-cell factor/lymphoid enhancing factor. ${ }^{31,32}$ Because miR-135a-5p imposed $G_{1}$ arrest of the glioblastoma cells, cyclin D1 and c-Myc levels were detected, and they were found to be dramatically decreased in the miR-135a-5p-overexpressing sub-cell lines. AKT is an important modulator of $\beta$-catenin nuclear translocation. $^{32,33}$ Activated AKT (phosphorylated AKT) phosphorylates and inactivates glycogen synthase kinase, which captures cytoplasmic $\beta$-catenin and induces its proteasome degradation by phosphorylation and ubiquitination, thus facilitating $\beta$-catenin nuclear translocation. ${ }^{32-34}$ The $\beta$-catenin in nuclei then promotes the transcription of cyclinD1 and $c-M y c$ genes. ${ }^{32,33}$ This study shows that the inhibitory effects of miR-135a-5p on the ATK phosphorylation, cyclin D1, and c-Myc expressions, cell cycle progression, and proliferation of glioma cells may be reversed completely by TRAF5 overexpression and mimicked perfectly by the specific siRNAs against TRAF5. In vivo experiments further verified that miR-135a-5p overexpression may inhibit xenograft tumor growth, reduce its cell density and Ki-67 index, and prevent animals from weight loss and prolong their survival. These findings demonstrate that miR-135a-5p suppresses the expressions of cyclin D1 and c-Myc by interdicting the TRAF5/AKT/ $\beta$ catenin pathway, thereby inhibiting the $\mathrm{G}_{1} / \mathrm{S}$-phase transition and proliferation of glioma cells (Figure 8E).

In summary, the expression changes of miR-135a-5p and TRAF5 in gliomas of various grades were shown, and their associations with the patients' outcome were unveiled. This work also demonstrated the inhibitory effect of miR-135a$5 \mathrm{p}$ on glioma cell proliferation and outlined the underlying molecular pathway. These results highlight the usefulness of miR-135a-5p and TRAF5 in the grading, molecular subclassification, and prognosis assessment of gliomas; they suggest that miR-135a-5p and TRAF5 are potentially candidates of malignant glioma therapy.

\section{Supplemental Data}

Supplemental material for this article can be found at https://doi.org/10.1016/j.ajpath.2018.08.019.

\section{References}

1. Ricard D, Idbaih A, Ducray F, Lahutte M, Hoang-Xuan K, Delattre JY: Primary brain tumours in adults. Lancet 2012, 379:1984-1996

2. Louis DN, Perry A, Reifenberger G, von Deimling A, FigarellaBranger D, Cavenee WK, Ohgaki H, Wiestler OD, Kleihues P, Ellison DW: The 2016 World Health Organization classification of tumors of the central nervous system: a summary. Acta Neuropathol 2016, 131:803-820

3. Wen PY, Kesari S: Malignant gliomas in adults. N Engl J Med 2008, 359:492-507

4. Marosi C, Preusser M: Milestones of the last 10 years: CNS cancer. Memo 2017, 10:18-21

5. Brennan CW, Verhaak RG, McKenna A, Campos B, Noushmehr H, Salama SR, et al: The somatic genomic landscape of glioblastoma. Cell 2013, 155:462-477

6. Li H, Yu L, Liu J, Bian X, Shi C, Sun C, Zhou X, Wen Y, Hua D, Zhao S, Ren L, An T, Luo W, Wang Q, Yu S: miR-320a functions as a suppressor for gliomas by targeting SND1 and beta-catenin, and predicts the prognosis of patients. Oncotarget 2017, 8:19723-19737

7. Shi C, Ren L, Sun C, Yu L, Bian X, Zhou X, Wen Y, Hua D, Zhao S, Luo W, Wang R, Rao C, Wang Q, Yu S: miR-29a/b/c function as invasion suppressors for gliomas by targeting CDC42 and predict the prognosis of patients. Br J Cancer 2017, 117:1036-1047

8. Szopa W, Burley TA, Kramer-Marek G, Kaspera W: Diagnostic and therapeutic biomarkers in glioblastoma: current status and future perspectives. Biomed Res Int 2017, 2017:8013575

9. Lu J, Getz G, Miska EA, Alvarez-Saavedra E, Lamb J, Peck D, SweetCordero A, Ebert BL, Mak RH, Ferrando AA, Downing JR, Jacks T, Horvitz HR, Golub TR: MicroRNA expression profiles classify human cancers. Nature 2005, 435:834-838

10. Calin GA, Croce CM: MicroRNA-cancer connection: the beginning of a new tale. Cancer Res 2006, 66:7390-7394

11. Croce CM: miRNAs in the spotlight: understanding cancer gene dependency. Nat Med 2011, 17:935-936 
12. Barciszewska AM: MicroRNAs as efficient biomarkers in high-grade gliomas. Folia Neuropathol 2016, 54:369-374

13. Shi H, Ji Y, Zhang D, Liu Y, Fang P: miR-135a inhibits migration and invasion and regulates EMT-related marker genes by targeting KLF8 in lung cancer cells. Biochem Biophys Res Commun 2015, 465: $125-130$

14. Cheng Z, Liu F, Zhang H, Li X, Li Y, Li J, Cao Y, Cao L, Li F: miR135a inhibits tumor metastasis and angiogenesis by targeting FAK pathway. Oncotarget 2017, 8:31153-31168

15. Dang Z, Xu WH, Lu P, Wu N, Liu J, Ruan B, Zhou L, Song WJ, Dou KF: MicroRNA-135a inhibits cell proliferation by targeting Bmil in pancreatic ductal adenocarcinoma. Int J Biol Sci 2014, 10:733-745

16. Xu B, Tao T, Wang Y, Fang F, Huang Y, Chen S, Zhu W, Chen M: hsa-miR-135a-1 inhibits prostate cancer cell growth and migration by targeting EGFR. Tumour Biol 2016, 37:14141-14151

17. Nagel R, le Sage C, Diosdado B, van der Waal M, Oude Vrielink JA, Bolijn A, Meijer GA, Agami R: Regulation of the adenomatous polyposis coli gene by the miR-135 family in colorectal cancer. Cancer Res 2008, 68:5795-5802

18. Li M, Long C, Yang G, Luo Y, Du H: miR-26b inhibits melanoma cell proliferation and enhances apoptosis by suppressing TRAF5-mediated MAPK activation. Biochem Biophys Res Commun 2016, 471: 361-367

19. Tao T, Cheng C, Ji Y, Xu G, Zhang J, Zhang L, Shen A: Numbl inhibits glioma cell migration and invasion by suppressing TRAF5-mediated NF-kappaB activation. Mol Biol Cell 2012, 23: $2635-2644$

20. Wang B, Zhao H, Zhao L, Zhang Y, Wan Q, Shen Y, Bu X, Wan M, Shen C: Up-regulation of OLR1 expression by TBC1D3 through activation of TNFalpha/NF-kappaB pathway promotes the migration of human breast cancer cells. Cancer Lett 2017, 408:60-70

21. Liu J, Xu J, Li H, Sun C, Yu L, Li Y, Shi C, Zhou X, Bian X, Ping Y, Wen Y, Zhao S, Xu H, Ren L, An T, Wang Q, Yu S: miR-146b-5p functions as a tumor suppressor by targeting TRAF6 and predicts the prognosis of human gliomas. Oncotarget 2015, 6:29129-29142

22. Li Y, Wang Y, Yu L, Sun C, Cheng D, Yu S, Wang Q, Yan Y, Kang C, Jin S, An T, Shi C, Xu J, Wei C, Liu J, Sun J, Wen Y, Zhao S, Kong Y: miR-146b-5p inhibits glioma migration and invasion by targeting MMP16. Cancer Lett 2013, 339:260-269

23. Liang Y, Diehn M, Watson N, Bollen AW, Aldape KD, Nicholas MK, Lamborn KR, Berger MS, Botstein D, Brown PO, Israel MA: Gene expression profiling reveals molecularly and clinically distinct subtypes of glioblastoma multiforme. Proc Natl Acad Sci U S A 2005, 102:5814-5819

24. Bredel M, Bredel C, Juric D, Harsh GR, Vogel H, Recht LD, Sikic BI: Functional network analysis reveals extended gliomagenesis pathway maps and three novel MYC-interacting genes in human gliomas. Cancer Res 2005, 65:8679-8689

25. Shi Y, Chen C, Yu SZ, Liu Q, Rao J, Zhang HR, Xiao HL, Fu TW, Long $\mathrm{H}$, He ZC, Zhou K, Yao XH, Cui YH, Zhang X, Ping YF, Bian XW: miR-663 suppresses oncogenic function of CXCR4 in glioblastoma. Clin Cancer Res 2015, 21:4004-4013

26. Furnari FB, Fenton T, Bachoo RM, Mukasa A, Stommel JM, Stegh A, Hahn WC, Ligon KL, Louis DN, Brennan C, Chin L, DePinho RA, Cavenee WK: Malignant astrocytic glioma: genetics, biology, and paths to treatment. Genes Dev 2007, 21:2683-2710

27. Walsh MC, Lee J, Choi Y: Tumor necrosis factor receptor-associated factor 6 (TRAF6) regulation of development, function, and homeostasis of the immune system. Immunol Rev 2015, 266:72-92

28. Li W, Peng C, Lee MH, Lim D, Zhu F, Fu Y, Yang G, Sheng Y, Xiao L, Dong X, Ma W, Bode AM, Cao Y, Dong Z: TRAF4 is a critical molecule for Akt activation in lung cancer. Cancer Res 2013, 73:6938-6950

29. Liu W, Qi Y, Liu L, Tang Y, Wei J, Zhou L: Suppression of tumor cell proliferation by quinine via the inhibition of the tumor necrosis factor receptor-associated factor 6-AKT interaction. Mol Med Rep 2016, 14 : 2171-2179

30. Mahajan K, Mahajan NP: PI3K-independent AKT activation in cancers: a treasure trove for novel therapeutics. J Cell Physiol 2012, 227 : $3178-3184$

31. Shtutman M, Zhurinsky J, Simcha I, Albanese C, D'Amico M, Pestell R, Ben-Ze'ev A: The cyclin D1 gene is a target of the betacatenin/LEF-1 pathway. Proc Natl Acad Sci U S A 1999, 96: $5522-5527$

32. McCubrey JA, Steelman LS, Bertrand FE, Davis NM, Abrams SL, Montalto G, D'Assoro AB, Libra M, Nicoletti F, Maestro R, Basecke J, Cocco L, Cervello M, Martelli AM: Multifaceted roles of GSK-3 and Wnt/beta-catenin in hematopoiesis and leukemogenesis: opportunities for therapeutic intervention. Leukemia 2014, 28:15-33

33. Freyberg Z, Ferrando SJ, Javitch JA: Roles of the Akt/GSK-3 and Wnt signaling pathways in schizophrenia and antipsychotic drug action. Am J Psychiatry 2010, 167:388-396

34. Tauriello DV, Maurice MM: The various roles of ubiquitin in Wnt pathway regulation. Cell Cycle 2010, 9:3700-3709 